\title{
Automated surface inspection for statistical textures
}

\author{
D. M. Tsai and T. Y. Huang \\ Machine Vision Lab. \\ Department of Industrial Engineering and Management \\ Yuan-Ze University, Chung-Li, Taiwan, R.O.C. \\ E-mail: iedmtsai@ saturn.yzu.edu.tw
}

\begin{abstract}
In this paper we present a global approach for the automatic inspection of defects in randomly textured surfaces which arise in sandpaper, castings, leather, and many industrial materials. The proposed method does not rely on local features of textures. It is based on a global image reconstruction scheme using the Fourier transform. Since a statistical texture has the surface of random pattern, the spread of frequency components in the power spectrum space is isotropic and forms the shape approximate to a circle. By finding an adequate radius in the spectrum space, and setting the frequency components outside the selected circle to zero, we can remove the periodic, repetitive patterns of any statistical textures using the inverse Fourier transform. In the restored image, the homogeneous region in the original image will have an approximately uniform gray level, and yet the defective region will be distinctly preserved. This converts the difficult defect detection in textured images into a simple thresholding problem in nontextured images. The experimental results from a variety of real statistical textures have shown the efficacy of the proposed method.
\end{abstract}

Keywords: Surface inspection; Defect detection; Statistical textures; Fourier transform; Image reconstruction 


\section{Introduction}

Image analysis techniques are being increasingly used to automate industrial inspection. The manual activity of inspection could be subjective and highly dependent on the experience of human personnel. In this study, we use machine vision for defect detection in randomly textured surfaces.

In automatic surface inspection, small defects that appear as local anomalies embedded in homogeneous textures must be detected. Textures are generally classified into two major types: structural and statistical [1]. Structural textures are those that are composed of repetitions of some basic texture primitives, such as oriented lines, with deterministic rules of displacement. This type of textures arises in textile fabrics, machined surfaces, printed wafers, etc. Statistical textures cannot be described with primitives and deterministic rules of displacement. The spatial distribution of gray levels in such textured images is rather stochastic. Sandpaper, leather, and many magnified metallic surfaces, for instance, fall in this category. The inspection task in this paper is classified as qualitative inspection [2] which involves detecting novel but obviously faulty items such as scratches, cracks, stains, wear and other ill-defined faults. Many of these unanticipated defects are small in size, and cannot be described by explicit measures, making automatic defect detection difficult.

Most of the defect detection systems are focused on nontextured surfaces such as glass panel [3], sheet steel [4] and uniform web materials [5] using simple thresholding or edge detection techniques. Defects in these images can be easily detected because they commonly have distinctly measured values with respect to those of the uniform background. Automatic visual inspection techniques for 
textured images generally compute a set of textural features in the spatial domain or in the spectral domain, and then search for significant local deviations in the feature values using various classifiers. In spatial-domain approaches, the commonly used features are the second-order statistics derived from spatial gray-level co-occurrence matrices [6]. They have been applied to wood inspection [7], carpet wear assessment [8], roughness measurement of machined surfaces [9], and surface defect detection [10].

In digital images, typical noise processes tend to dramatically alter local spatial variation of intensity while having relatively uniform representation in the spectral domain [11]. Spectral-domain features are generally less sensitive to noise than spatial-domain features. In spectral-domain approaches, the textural features are generally derived from the Fourier transform [12, 13], Gabor transform [14, 15], and wavelet transform [16]. Liu and Jernigan [11] reviewed a set of 28 textural features extracted in the Fourier spectrum for texture analysis. Escofet et al. [17] used the angular correlation of the Fourier spectra to evaluate fabric web resistance to abrasion. Chan and Pang [18] used the Fourier analysis for fabric defect detection. Seven textural features extracted from the vertical and horizontal frequency components in the Fourier spectrum are used to discriminate four defect types including double yarn, missing yarn, webs and yarn densities.

In the last decade, the multichannel Gabor filters $[14,19]$ are well recognized as a joint spatial/spatial-frequency representation for analyzing textured images with highly specific frequency and orientation characteristics. This technique extracts features by filtering the textured image with a set of Gabor filter banks characterized by the frequency, the orientation of the sinusoid, and the scale of the window function. 
Clausi and Jernigan [20] compared various Gabor filter implementations for texture analysis. They found that using the Gabor filter magnitude response given a frequency bandwidth and spacing of one octave and orientation bandwidth and spacing of 30 degrees generated preferred results. The Gabor filtering techniques have been applied to the inspection of wooden surfaces [21], granite [22], steel surfaces [23], and textile fabrics [24].

In the recent past, multiresolution decomposition schemes based on wavelet transforms have received considerable attention as alternatives for the extraction of textural features. The multiresolution wavelet representation allows an image to be decomposed into a hierarchy of localized subimages at different spatial frequencies [25]. It divides the 2D frequency spectrum of an image into a lowpass (smooth) subimage and a set of highpass (detail) subimages. The textural features are then extracted from the decomposed subimages in different frequency channels and different resolution levels. Chen and Kuo [26] proposed a tree-structured wavelet transform for texture classification. They used an energy criterion to select the subimages for decomposition. A set of textural features is derived from the energy values of dominant channels, and distance measures are then employed to discriminate texture classes. Laine and Fan [27] used both energy and entropy metrics of each decomposed wavelet packet as textural features. The number of features is numerous. It involves 17 features of standard wavelet decomposition, and 341 features of a complete set of wavelet packets. A two-layer neural network then uses those features as input for texture classification. Lambert and Bock [28] also proposed a feature extraction approach for texture defect detection. The textural features are derived from the coefficients of wavelet packet decomposition. Neural network and Bayes classifiers are used to evaluate the feature vector. The 
wavelet-based feature extraction methods have been applied to the inspection of industrial materials such as LSI wafers [29], cold rolled strips [30], and woven fabrics [31].

The texture analysis and defect detection methods aforementioned are generally based on the extraction of textural features in the spatial and spectral domains. The most difficult task of this approach is to select adequate textural features which most completely embody information of the texture under study. A set of features that is an optimal representation of a specific texture could be completely useless for other texture patterns. There is no straightforward manner to judge the appropriate features to use. Therefore, the selection of an adequate feature set for a new texture in the training process could be very time consuming and requires the knowledge of human experts. An important problem arisen in feature extraction approaches is that textures characterized by a vector of multiple features results in high dimensionality. This calls for sophisticated classifiers such as Bayes [32], maximum likelihood [33], Markov random field [34], and neural networks [35] to discriminate texture classes.

The feature-extraction methods aforementioned for texture analysis are generally based on local techniques. Tsai and Hsieh [36] proposed a global approach based on a Fourier image reconstruction scheme for inspecting surface defects in oriented textures. The specific structural textures in their study must be a homogeneous pattern that consists of an arrangement primarily of line structures appearing periodically on the surface. Textile fabrics and milled surfaces are two examples that fall in the class of oriented textures. Their method does not depend on local features of textures. The line pattern of any oriented texture in the spatial-domain image can be easily removed by detecting the high-energy frequency components in 
the Fourier spectrum, setting them to zero, and finally back-transforming to a spatial-domain image. The transform of periodic lines of a specific orientation $\theta$ in the spatial domain image appears a line orthogonal to $\theta$ in the Fourier domain image. The line associated with high-energy frequency components in the Fourier spectrum is detected using the Hough transform. In the restored image of a textured surface, the regular region with periodic lines in the original image will have an approximately uniform gray level, whereas the defective region will be distinctly preserved. Their approach has focused on oriented textures, and has worked successfully on a variety of real samples including textile fabrics, machined surfaces and natural wood. However, it cannot be directly extended to the inspection of statistical textures such as sandpaper and leather surfaces since statistical texture patterns are isotropic, and high-energy frequency components will not distribute along lines in the Fourier spectrum.

In this paper, we also adopt the global image reconstruction scheme using the Fourier transform for surface defect detection, but mainly focus on statistical textures with isotropic patterns. This paper fulfills the Fourier image reconstruction scheme for defect detection in both structural and statistical textures. For a statistical texture, the spread of high-energy frequency components in the Fourier spectrum image is isotropic and forms the shape approximate to a circle. The power magnitude is generally decreased as the frequency components are away from the central component in the Fourier spectrum image. By finding an adequate radius of a circle with origin at the center of the Fourier spectrum image, and setting the frequency components outside the selected circle to zero, we can remove the periodic, repetitive patterns of any statistical textures and preserve only local anomalies using the inverse Fourier transform. The radius to be selected in the Fourier spectrum image is based 
on the curvature analysis of average power magnitude in each ring of varying radius. In the restored image, the homogenous region in the original textured image will have an approximately uniform gray level, and the defective region will be distinctly enhanced. A simple thresholding process can then be used to discriminate between defective regions and homogeneous regions in the restored image. This converts the difficult defect detection problem in complicated textured images into a simple thresholding problem in nontextured images.

This paper is organized as follows: Section 2 first discusses the Fourier transform and the properties of statistical textures in the Fourier spectrum image. Then the curvature analysis used to determine the best radius for back-transforming of the spectrum image is described. Section 3 presents the experimental results on a variety of real statistical textures including sandpaper, castings, leather and carpets. The paper is concluded in Section 4.

\section{Removal of periodic patterns}

\subsection{The Fourier spectra of statistical textures}

In this paper we use machine vision to solve the problem of detecting small surface defects which appear as local anomalies embedded in homogeneous, statistical textures. The Fourier-based image reconstruction technique is used to remove the repetitive, periodic patterns in randomly textured images. We are then able to identify anomalies in the restored image. The Fourier transform (FT) has the desirable properties of noise-immunity and enhancement of periodic features. The FT characterizes the textured image in terms of frequency components. The 
periodically occurring features can be observed from the magnitude of frequency components. These global texture patterns are easily distinguishable as concentration of high-energy bursts in the spectrum.

Let $f(x, y)$ be the gray level at pixel coordinates $(x, y)$ in the original image of size $N \times N$. The discrete 2D FT of $f(x, y)$ is given by

$$
F(u, v)=\frac{1}{N} \sum_{x=-\frac{N}{2}}^{\frac{N}{2}} \sum_{y=-\frac{N}{2}}^{\frac{N}{2}} f(x, y) \cdot \exp [-j \cdot 2 \pi(u x+v y) / N]
$$

for frequency variables $u, v=-N / 2,-N / 2+1, \ldots, N / 2-1, N / 2$. The discrete 2D FT can be expressed in the separable forms with two 1D FTs, and obtained efficiently using the last FT algorithm [37], given that $N$ is a power of two. The FT is generally complex; that is

$$
F(u, v)=R(u, v)+j \cdot I(u, v)
$$

where $R(u, v)$ and $I(u, v)$ are the real and imaginary components of $F(u, v)$, i.e.

$$
\begin{aligned}
& R(u, v)=\frac{1}{N} \sum_{x=-\frac{N}{2}}^{\frac{N}{2}} \sum_{y=-\frac{N}{2}}^{\frac{N}{2}} f(x, y) \cdot \cos [2 \pi(u x+v y) / N] \\
& I(u, v)=-\frac{1}{N} \sum_{x=-\frac{N}{2}}^{\frac{N}{2}} \sum_{y=-\frac{N}{2}}^{\frac{N}{2}} f(x, y) \cdot \sin [2 \pi(u x+v y) / N]
\end{aligned}
$$

The power spectrum $P(u, v)$ of image $f(x, y)$ is defined by

$$
P(u, v)=|F(u, v)|^{2}=[R(u, v)]^{2}+[I(u, v)]^{2}
$$

Since a statistical texture contains isotropic structure, the high-energy 
components in the spectrum image are also spread omnidirectionally and form the shape of a circular disk. A fine textured surface results in a larger spread radius, whereas a coarse one yields a smaller spread radius in the Fourier spectrum image. The magnitude of power for frequency components away from the central component in the spectrum image falls off rapidly to approximately zero for coarse textured surfaces, and is reduced gradually for fine textured surfaces. Therefore, various radii of the spread disk with origin at the center of the Fourier spectrum image contain different levels of the detail information in the textured image. Figures 1(a)-(c) show the surface images of three castings with varied roughness classes. Figures 1(d)-(f) visually show the power spectra $P(u, v)$ of the surface images as an intensity function, where brightness is proportional to the power magnitude. Figures 1(g)-(i) present the plots of the power spectra in $3 \mathrm{D}$ perspective. It can be seen from Figure 1 that a fine cast surface generates a larger spread radius, but a coarse one yields a smaller radius in the Fourier spectrum.

Figure 2(a) displays a faultless surface image of sandpaper, and Figure 2(b) is a defective version of the sandpaper surface. Figures 2(c) and 2(d) illustrate the power spectra as an intensity function, and Figures 2(e) and 2(f) present the plots of the power spectra in $3 \mathrm{D}$ perspective. It can be observed from Figures $2(\mathrm{e})$ and $2(\mathrm{f})$ that high-energy frequency components are distributed around the center in the Fourier spectrum. The spread radius associated with the defective surface in Figure 2(b) is visibly larger than the one of the faultless surface in Figure 2(a). It indicates that the regular textured-pattern can be blurred, and yet the anomaly can be preserved by restoring only the frequency components inside a circle of properly selected radius.

Denote $r_{\max }$ by the selected radius of the circular disk in the Fourier spectrum. 
Let $r_{\max }=12$ for the faultless surface (Figures 2(a) and 2(c)), and $r_{\max }=21$ for the defective surface (Figures 2(b) and 2(d)), which are selected to reconstruct the textured images. Figures 3(c) and 3(d) show the reconstruction results by setting all frequency components inside the selected circle to zero, i.e.

$$
F(u, v)=0, \quad \forall \quad u^{2}+v^{2} \leq r_{\max }^{2}
$$

where the center coordinates $(u, v)=(0,0)$ in the Fourier spectrum image. The restored images in Figure 3 show that the periodic, repetitive pattern is not cleanly removed and the anomaly is not well preserved.

Figures 4(c) and 4(d) show the reconstruction results by setting all frequency components outside the selected circle to zero, i.e.

$$
F(u, v)=0, \quad \forall \quad u^{2}+v^{2}>r_{\max }^{2}
$$

The restored images in Figure 4 show that the textures are severely blurred, but the periodic, repetitive patterns are not cleanly removed. To sufficiently remove the periodic textured-pattern and distinctly preserve anomalies in the restored image, all frequency components outside the selected circle of radius $r_{\max }$ along with the central component at $(0,0)$ are set to zero, i.e.

$$
F(u, v)=0, \quad \forall \quad u^{2}+v^{2}>r_{\max }^{2} \text { and }(u, v)=(0,0)
$$

Figures 5(c) and 5(d) show the reconstruction results with such setting. It can be seen from Figure 5 that the periodic, repetitive patterns are nearly eliminated in both faultless and defective surfaces. The faultless surface results in an approximately uniform image, and the anomaly in the defective surface is notably separated in the 
restored image.

Figures 6(a1)-(a4) and 6(b1)-(b4) show the reconstruction results from the circles of radii 5, 10, 20 and 40 pixels for the faultless surface in Figure 2(a), and the defective surface in Figure 2(b), respectively. For faultless surfaces, the smaller the radius is used, the better the gray-level uniformity is obtained in the restored images since more high frequency energy is removed. However, for defective surfaces, an overly small radius may also severely blur the anomaly in the restored images, as seen in Figure 6(b1). Therefore, the success of the global image reconstruction scheme for textured surface inspection relies on the selection of an appropriate radius that can sufficiently remove periodic textured-patterns, and yet distinctly preserve the anomalies in the restored images. In the following subsection, we propose a curvature analysis procedure to automatically determine the appropriate radius for image reconstruction.

\subsection{Selection of radius $r_{\max }$ for image reconstruction}

Denote $E(r)$ by the average power of the ring with radius $r$,

$$
E(r)=\frac{1}{n_{r}} \sum_{u^{2}+v^{2}=r^{2}} P(u, v), \quad r=0,1,2,3, \ldots, N / 2
$$

where $n_{r}$ is the total number of points on the ring of radius $r$, and $N$ is the image width. While the ring is grown outwards from the center $(0,0)$ of the Fourier spectrum, the average power magnitude generally decreases rapidly. Figure 7 depicts the plot of average power $E(r)$ with respect to varying radius $r$ for Figures 2(a) and 2(b). Note that the $E(r)-r$ curves become flat soon after the radii $r_{\max }$ 
which visibly give the maximum curvatures for individual faultless and defective textures. The maximum curvatures are obtained at $r_{\max }=12$ for the faultless texture in Figure 2(a), and at $r_{\max }=21$ for the defective texture in Figure 2(b). Both selected radii $r_{\max }$ have performed well for the detection of anomaly and removal of periodic patterns, as seen in Figure 5. Therefore, the best radius $r_{\max }$ used for image reconstruction is selected from the maximum curvature of the $E(r)-r$ curve. The selection procedure of radius $r_{\max }$ is given as follows:

Let $\psi(r)$ and $\kappa(r)$ be the respective tangent and curvature at radius $r$ in the $E(r)-r$ curve,

$$
\begin{aligned}
& \psi(r)=\tan ^{-1}\left[\frac{\Delta E(r)}{s}\right]=\tan ^{-1}\left[\frac{E(r)-E(r-s)}{s}\right] \\
& \kappa(r)=\frac{\Delta \psi(r)}{\Delta s}=\frac{\psi(r)-\psi(r-s)}{s}, \quad r=0,1,2,3, \ldots, N / 2 \\
& r_{\max }=\arg \left\{\max _{r} \kappa(r)\right\}
\end{aligned}
$$

where $s$ is the length of support used to compute the first- and second-order differences. In this study, the length of support $s$ is set to 2 .

Once the best radius $r_{\max }$ is determined, the values of frequency components $F(u, v)$ are reassigned below.

$$
\hat{F}(u, v)=\left\{\begin{array}{l}
0, \text { if } u^{2}+v^{2}>r_{\max }^{2} \text { or }(u, v)=(0,0) \\
F(u, v), \text { otherwise }
\end{array}\right.
$$

After scanning all frequency components $(u, v)$ in the Fourier spectrum, we can reconstruct the image using the inverse Fourier transform. That is, 


$$
\hat{f}(x, y)=\frac{1}{N} \sum_{u=-\frac{N}{2}}^{\frac{N}{2}} \sum_{v=-\frac{N}{2}}^{\frac{N}{2}} \hat{F}(u, v) \cdot \exp [j \cdot 2 \pi(u x+v y) / N]
$$

Note that the restored image $\hat{f}(x, y)$ will be approximately a uniform gray-level image if it is a faultless image, as seen in Figure 5(c). It yields distinct gray values for pixels in defective regions, as shown in Figure 5(d). The intensity variation in homogeneous regions will be small, whereas the gray-level variation in defective regions will be huge with respect to the whole restored image. Therefore, we can use the simple statistical process control principle to set up the control limits for distinguishing defects from the uniform background in the restored image. The upper and lower control limits for intensity variation in the restored image are given by

$$
\mu_{\hat{f}} \pm c \sigma_{\hat{f}}
$$

where $\mu_{\hat{f}}$ and $\sigma_{\hat{f}}$ are the mean and standard deviation of gray levels in the restored image $\hat{f}(x, y)$ of size $N \times N$, i.e.

$$
\begin{aligned}
& \mu_{\hat{f}}=\frac{1}{N^{2}} \sum_{x} \sum_{y} \hat{f}(x, y) \\
& \sigma_{\hat{f}}=\left\{\frac{1}{N^{2}-1} \sum_{x} \sum_{y}\left[\hat{f}(x, y)-\mu_{\hat{f}}\right]^{2}\right\}^{1 / 2}
\end{aligned}
$$

$c$ is a control constant. Since the gray levels of pixels in defective regions are distinctly different from those in regular regions in the restored image, a large $c$ value of 5 is used in this study. It can sufficiently eliminate random noise for faultless surfaces, and yet well segment the anomalies for defective surfaces in the 
restored images. If a pixel with the gray level falls within the control limits, the pixel is classified as a homogeneous element. Otherwise, it is classified as a defective element.

\section{Experiments and discussion}

In this section, we present experimental results on a variety of statistical textures including sandpaper, casting, leather and carpet to evaluate the performance of the proposed defect detection method. The algorithms are implemented on a personal computer using the $\mathrm{C}++$ language. The image is $256 \times 256$ pixels wide with 8-bit gray levels.

The proposed method does not require prestored texture images for reference, or any texture information of faultless samples. All test samples in the experiment use the support length $s=2$ for curvature measurement in the $E(r)-r$ curve, and $c=5$ for the control limits.

\subsection{Sensitivity of the selected radius $r_{\max }$}

In this study, the best radius $r_{\max }$ used for image reconstruction is selected based on the maximum curvature in the $E(r)-r$ curve. A faultless leather surface and a defective one, as shown in Figures 8(a) and 8(b), are used to evaluate the sensitivity of minor changes in $r_{\max }$ value. Figures 8(c) and 8(d) show the Fourier spectra as an intensity function, and Figure 8(e) depicts the $E(r)-r$ curves of the two test images. Figures 9(a1)-(a5) present the reconstruction results from radii of 7 , 9, 11, 13 and 25 pixels for the faultless texture in Figure 8(a), in which $r_{\max }=11$ 
gives the maximum curvature. Figures 9(b1)-(b5) show the reconstruction results from radii of 10, 16, 18, 20 and 25 pixels for the defective texture in Figure 8(b), in which $r_{\max }=18$ gives the maximum curvature. From Figure 9, we found that the radii in the neighborhood of the selected $r_{\max }$ generate similar reconstruction results. It is not crucial for the selection of a precise $r_{\max }$ value. The image reconstruction process can tolerate minor change of the selected radius $r_{\max }$.

\subsection{Experimental results}

The proposed method in this study is based on a global image reconstruction scheme for defect detection. It is insensitive to changes in the illumination intensity. Figure 10(a1) shows a defective leather surface under regular lighting condition. Figures 10(b1) and 10(c1) are underexposed and overexposed versions of the leather image in Figure 10(a1). Figure 11 depicts the $E(r)-r$ curves of the leather images in three different illumination intensities. The selected radii $r_{\max }$ for Figures 10(a1)-(c1) are 18, 17 and 20 pixels, respectively. Figures 10(a2)-(c2) and (a3)-(c3) respectively present the Fourier power spectra and the restored images of the three leather images. Figures 10(a4)-(c4) show the defect detection results of Figures 10(a3)-(c3) as binary images, where pixels with gray levels falling outside the control limits are represented in black, and the ones falling within the control limits appear as white. The results reveal that the defects in all three leather images are correctly segmented in the binarized images, regardless of lighting changes.

In order to show the effect of changes in defect size, Figure 12(a)-(c) presents, respectively, defects in increasing sizes on fine sandpaper surfaces. Figure 13(a)-(c) show additional samples of varied defect sizes on coarse sandpaper surfaces that 
contain less regular patterns. It can be seen from the results in Figures 12 and 13 that the defects are well enhanced in the restored images, even though the defects shown in Figures 12(c) and 13(c) occupy most of the image.

A number of test samples including cast, wool material, tile, leather, carpet, cork surfaces, and coarse-grain sandpaper are also evaluated in this section to demonstrate the effectiveness of the proposed method. These samples present both faultless and varied defective images for comparison. Figures 14(a1) and (b1) illustrate a faultless cast surface, and a defective cast with scratches. The resulting binary images in Figures 14(a4) and (b4) show that the faultless surface is a uniform white image, and the scratches are well detected. Figures 15(a1) and (b1) present a faultless and a defective wool surfaces, respectively. The corresponding binary images in Figures 15(a4) and (b4) also show that the faultless surface is a uniform white image, and the subtle anomaly at the center of the original image is correctly detected. Figures 16(a1) presents a faultless tile surface that contains random dot pattern. The four detective spots in Figure 16(b1) are well detected in the resulting binary image, as seen in Figure 16(b4).

Figures 17(a1)-(c1) show a faultless image of leather, a defective leather surface with a non-linear defect of wear, and a defective leather surface with a linearly structured scratch, respectively. Figures 17(a3) shows that the restored image of the faultless surface is almost a uniform gray level. Both nonlinear and linear types of defects in the leather surfaces are reliably detected in the resulting binary images, as seen in Figures 17(b4) and (c4). Figures 18-20 further demonstrate three complicated texture surfaces of carpet, cork and coarse-grain sandpaper that involve varied structural defects such as scratch, and ill-defined defects such as wear and 
shedding of fiber. The detection results show that the proposed method performs effectively for a variety of real statistical textures that contain various types of defects on homogeneous surfaces.

\section{Conclusions}

In this paper we have considered the problem of detecting local defects embedded in statistical textures. The proposed method does not rely on local textural features in a pixel-by-pixel basis. It is based on a global image reconstruction scheme using the Fourier transform. By properly selecting a circle of radius $r_{\max }$ with origin at the center of the Fourier spectrum image, and setting the frequency components at the center and outside the circle to zero, the global periodic, repetitive texture patterns can be effectively removed and local anomalies can be distinctly preserved in the restored image. A simple thresholding is therefore used to separate the defective regions from the uniform gray-level background in the restored image. The best radius $r_{\max }$ of the circle used for image reconstruction is automatically determined by the maximum curvature in the curve of average power with respect to varying radius. A minor change of $r_{\max }$ values between 1 and 5 pixels will not affect the reconstruction result. The proposed method in this paper is an unsupervised one since it does not require a prestored faultless image or any information of the reference texture. It makes the method insensitive to environmental changes such as lighting variation. The experimental results reveal that the proposed method is well suited for detecting defects in statistical textures that contain homogeneous patterns. The performance of the proposed method will be degraded gradually as the textured patterns become less regular. 
The proposed method in this study mainly focuses on the detection of defects in a textured image, but not the classification of defect types. Although the obtained segmentations from the proposed method could be blurred or not accurate in shape, it can still reliably detect the presence of defects in the textured image. For classification purpose, the proposed method can be used for fast localization of defects in the complicated texture. Local techniques can then be carried out in the neighborhood of the detected defect to extract features such as color and blobs of the defect. 


\section{References}

1. A. Pikaz, A. Averbuch, An efficient topological characterization of gray-levels textures using a multiresolution representation, Graphical Models and Image Processing 59 (1997) 1-17.

2. T. S. Newan, A. K. Jain, Survey of automated visual inspection, Computer Vision and Image Understanding 61 (1995) 231-262.

3. J. Wilder, Finding and evaluating defects in glass, in: H. Freeman (Ed.) Machine Vision for Inspection and Measurement, Academic Press, New York, 1989, p. 237.

4. J. Olsson, S. Gruber, Web process inspection using neural classification of scattering light, Proceedings of the IEEE International Conference on Industrial Electronics, Control, Instrumentation and Automation (IECON'92), 1992, pp. 1443-1448.

5. D. Brzakovic, N. Vujovic, Designing defect classification system: a case study, Pattern Recognition 29 (1996) 1401-1419.

6. R. M. Haralick, K. Shanmugam, I. Dinstein, Textural features for image classification, IEEE Trans. System, Man Cybernet. 3 (1973) 610-621.

7. R. W. Conners, C. W. McMillin, K. Lin, R. E. Vasquez-Espinosa, Identifying and locating surface defects in wood, IEEE Trans. Pattern Anal. Mach. Intell. PAMI-5 (1983) 573-583.

8. L. H. Siew, R. M. Hogdson, Texture measures for carpet wear assessment, IEEE Trans. Pattern Anal. Mach. Intell. 10 (1988) 92-105.

9. K. V. Ramana, B. Ramamoorthy, Statistical methods to compare the texture features of machined surfaces, Pattern Recognition 29 (1996) 1447-1459.

10. J. Iivarinen, Surface defect detection with histogram-based texture features, Proceedings of SPIE 4197, 2000, pp. 140-145.

11. S. -S. Liu, M. E. Jernigan, Texture analysis and discrimination in additive noise, Computer Vision, Graphics and Image Processing 49 (1990) 52-67.

12. H. Wechsler, Texture analysis: a survey, Signal Processing 2 (1980) 271-282.

13. R. Azencott, J. -P. Wang, L. Younes, Texture classification using windowed Fourier filters, IEEE Trans. Pattern Anal. Mach. Intell. 19 (1997) 148-153. 
14. J. G. Daugman, Uncertainty relation for resolution in space, spatial-frequency and orientation optimized by two-dimensional visual cortical filters, Journal of the Optical Society of America 2 (1985) 1160-1169.

15. M. Clark and A. C. Bovik, Texture segmentation using Gabor modulation/demodulation, Pattern Recognition Letters 6 (1987) 261-267.

16. S. G. Mallat, A theory for multiresolution signal decomposition: the wavelet representation, IEEE Trans. Pattern Anal. Mach. Intell. 11 (1989) 674-693.

17. J. Escofet, M. S. Millan, H. Abril, E. Torrecilla, Inspection of fabric resistance to abrasion by Fourier analysis, Proceedings of SPIE 3490, 1998, pp. 207-210.

18. C. -H. Chan, K. H. Pang, Fabric defect detection by Fourier analysis, IEEE Trans. Industry Applications 36 (2000) 1267-1276.

19. M. Porat, Y. Y. Zeevi, The generalized Gabor scheme of image representation in biological and machine vision, IEEE Trans. Pattern Anal. Mach. Intell. 10 (1988) 452-468.

20. D. A. Clausi, M. E. Jernigan, Designing Gabor filters for optimal texture separability, Pattern Recognition 33 (2000) 1835-1849.

21. W. Polzleitner, G. Schwingskakl, Quality classification of wooden surfaces using Gabor filters and genetic feature optimization, Proceedings of SPIE 3837, 1999, pp. 220-231.

22. G. Paschos, Fast color texture recognition using chromaticity moments, Pattern Recognition Letters 21 (2000) 837-841.

23. K. Wiltschi, A. Pinz, T. Lindeberg, Automatic assessment scheme for steel quality inspection, Machine Vision and Applications 12 (2000) 113-128.

24. A. Bodnarova, M. Bennamoun, S. J. Latham, Constrained minimisation approach to optimise Gabor filters for detecting flaws in woven textiles, Proceedings of the IEEE International Conference on Acoustics, Speech and Signal Processing 6 (2000) 3606-3609.

25. C. H. Chen, G. G. Lee, On digital mammogram segmentation and microcalcification detection using multiresolution wavelet analysis, Graphical Models and Image Processing 59 (1997) 349-364.

26. T. Chen, C. -C. J. Kuo, Texture analysis and classification with tree-structured wavelet transform, IEEE Trans. Image Processing 2 (1993) 429-441. 
27. A. Laine, J. Fan, Texture classification by wavelet packet signatures, IEEE Trans. Pattern Anal. Mach. Intell. 15 (1993) 1186-1191.

28. G. Lambert, F. Bock, Wavelet methods for texture defect detection, Proceedings of the IEEE International Conference on Image Processing 3, 1997, pp. 201-204.

29. K. Maruo, T. Shibata, T. Yamaguchi, M. Ichikawa, T. Ohmi, Automatic defect pattern detection on LSI wafers using image processing techniques, IEICE Transactions on Electronics E82-C (1999) 1003-1012.

30. C. S. Lee, C. -H. Choi, J. Y. Choi, S. H. Choi, Surface defect inspection of cold rolled strips with features based on adaptive wavelet packets, IEICE Trans. Inf. Syst. E80-D (1997) 594-604.

31. H. Sari-Sarraf, J. S. Goddard, Jr., Robust defect segmentation in woven fabrics, Proceedings of the IEEE Computer Society Conference on Computer Vision and Pattern Recognition, 1998, pp. 938-944.

32. L. M. Linnett, D. R. Carmichael, S. J. Clarke, Texture classification using a spatial-point process model, IEE Proceedings: Vision, Image and Signal Processing 14 (1995) 1-6.

33. F. S. Cohen, Maximum likelihood unsupervised textured image segmentation, CVGIP: Graphical Models Image Processing 54 (1992) 239-251.

34. F. S. Cohen, Z. Fan, M. A. Patel, Classification of rotated and scaled textured images using Gaussian Markov random field models, IEEE Trans. Pattern Anal. Mach. Intell. 13 (1991) 192-202.

35. M. M. Van Hulle, T. Tollenaere, A modular artificial neural network for texture processing, Neural Networks 6 (1993) 7-32.

36. D. - M. Tsai and C. $-\mathrm{Y}$ Hsieh, Automated surface inspection for directional textures, Image and Vision Computing 18 (1999) 49-62.

37. R. C. Gonzalez, R. E. Woods, Digital Image Processing, Addison-Wesley, Reading, MA, 1992. 


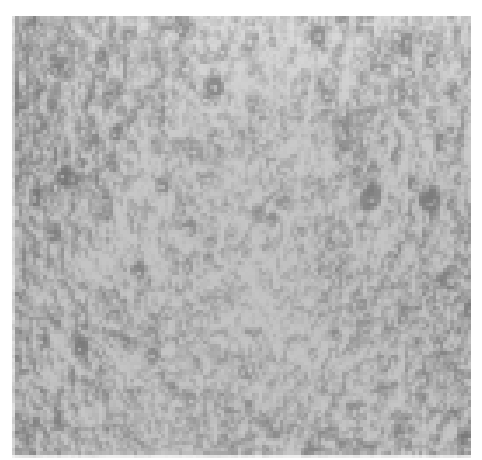

(a)

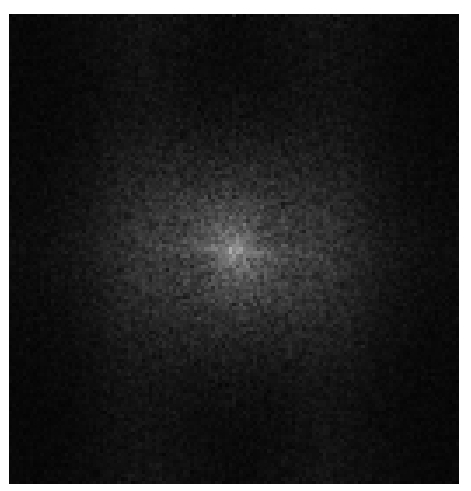

(d)

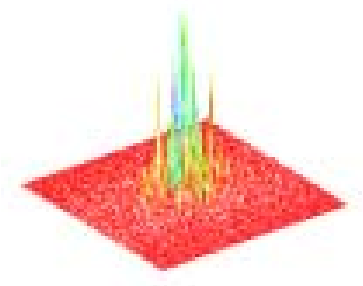

(g)

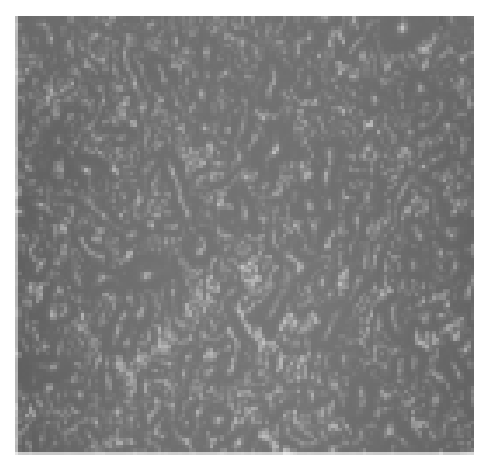

(b)

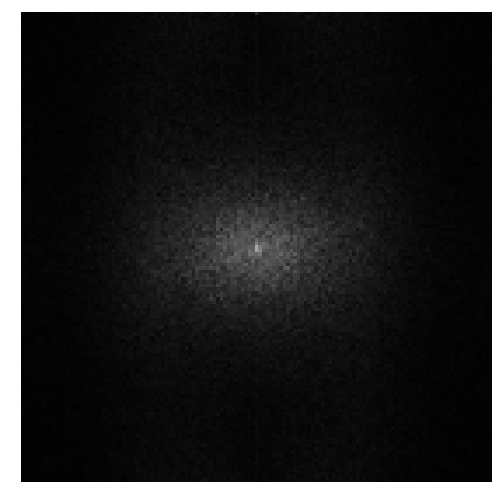

(e)

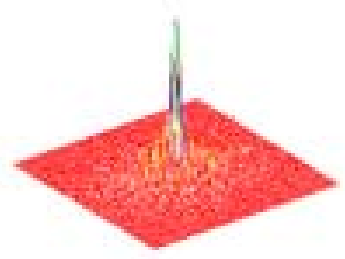

(h)

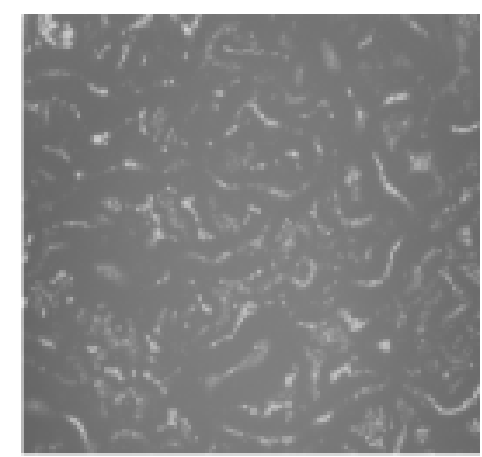

(c)

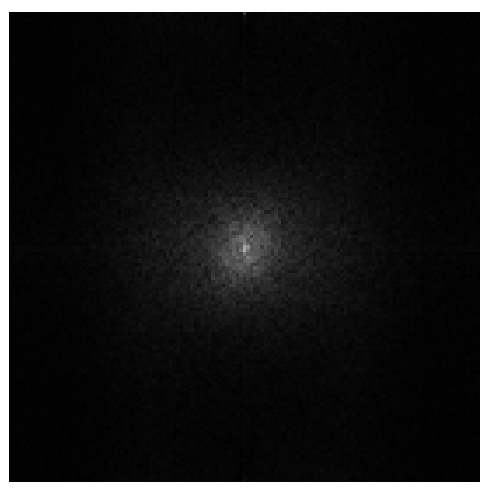

(f)

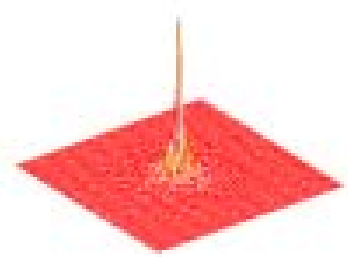

(i)

Figure 1. (a)-(c) Fine to coarse surface images of castings; (d)-(f) the corresponding power spectra displayed as an intensity function; (g)-(i) the corresponding power spectra in 3-D perspective. 


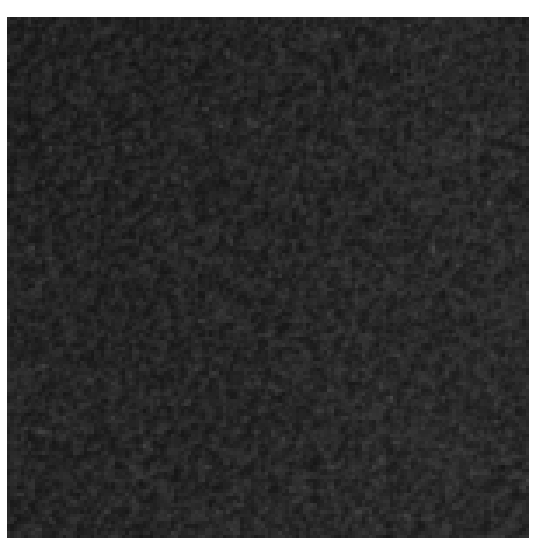

(a)

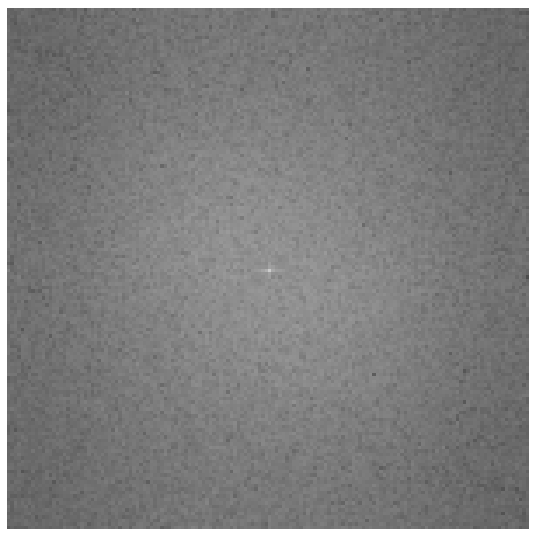

(c)

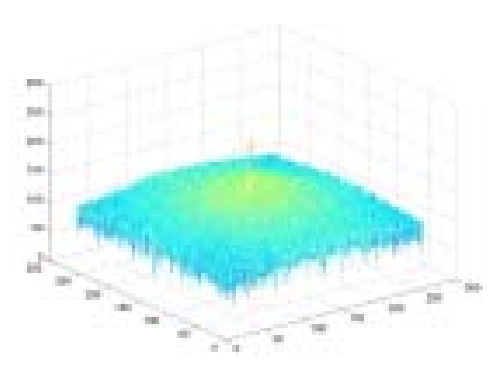

(e)

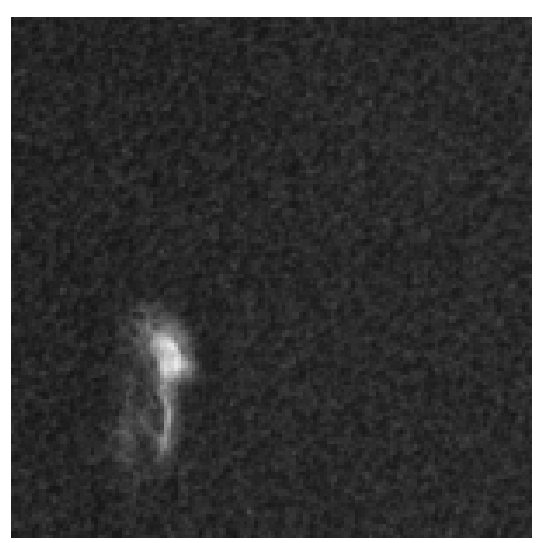

(b)

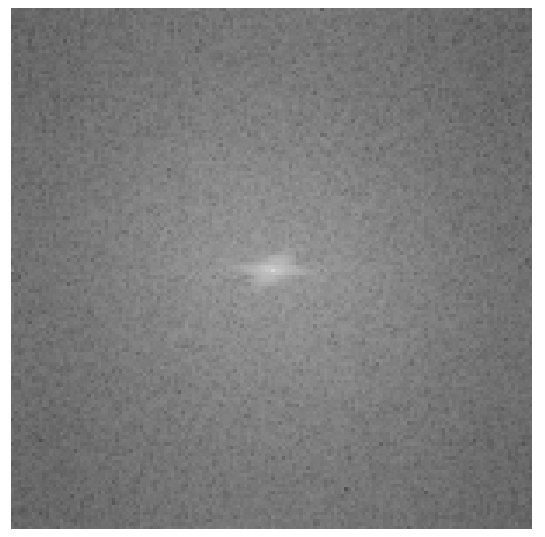

(d)

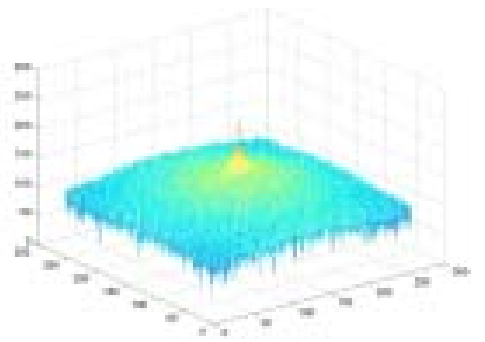

(f)

Figure 2. (a) A faultless surface image of sandpaper; (b) a defective sandpaper image; (c), (d) the respective Fourier domain images; (e), (f) the respective power spectra in 3-D perspective. 


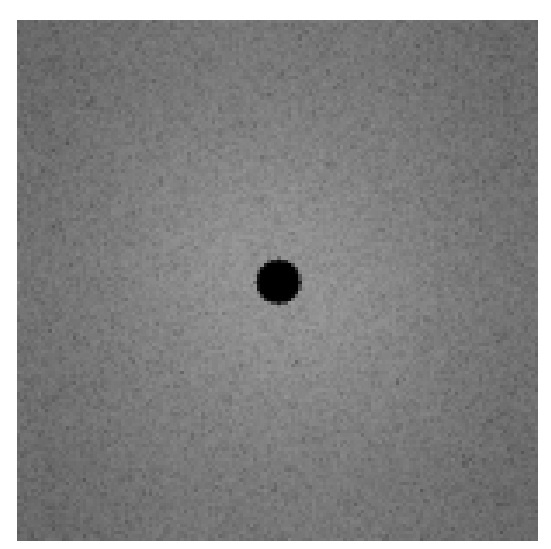

(a)

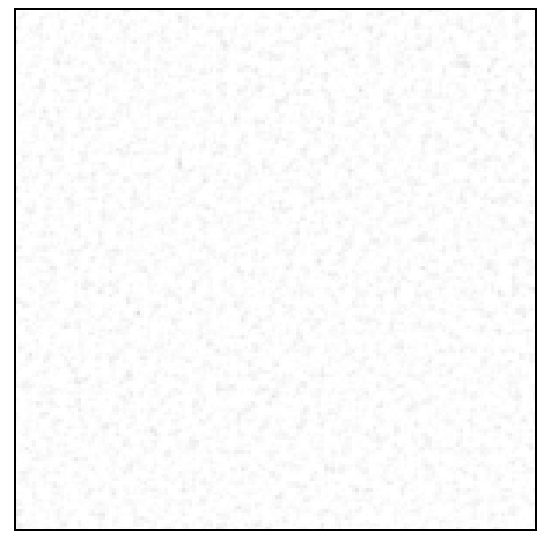

(c)

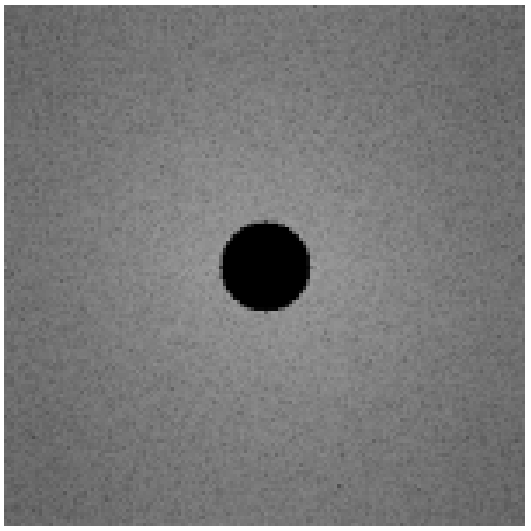

(b)

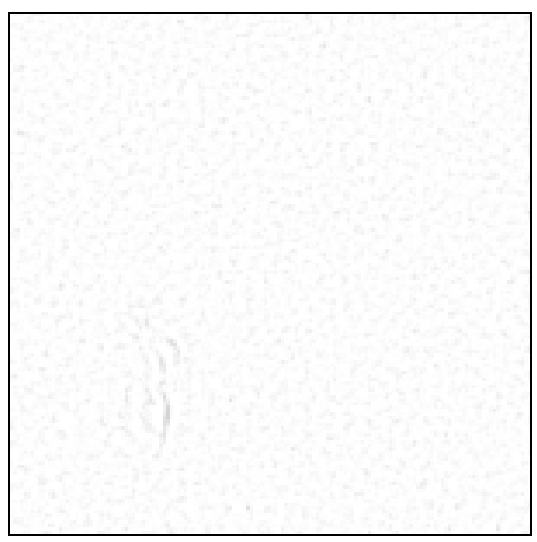

(d)

Figure 3. (a), (b) The respective Fourier domain images of Figures 2(a) and 2(b) with $F(u, v)=0$ (shown in blank) for all frequency components inside the circle of radius $r_{\max } ;(\mathrm{c}),(\mathrm{d})$ the respective restored images based on the power spectra in (a) and (b). 


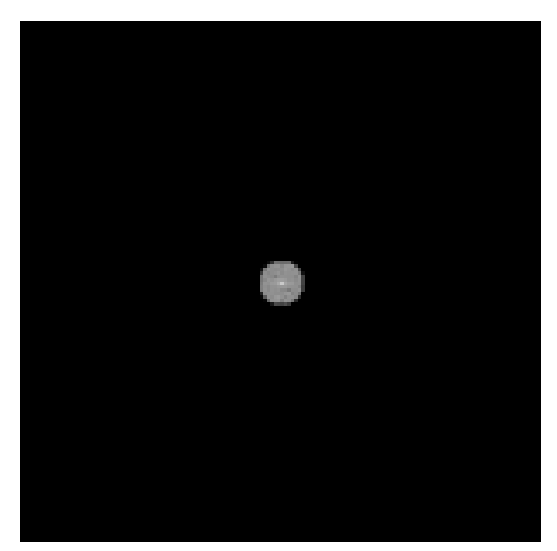

(a)

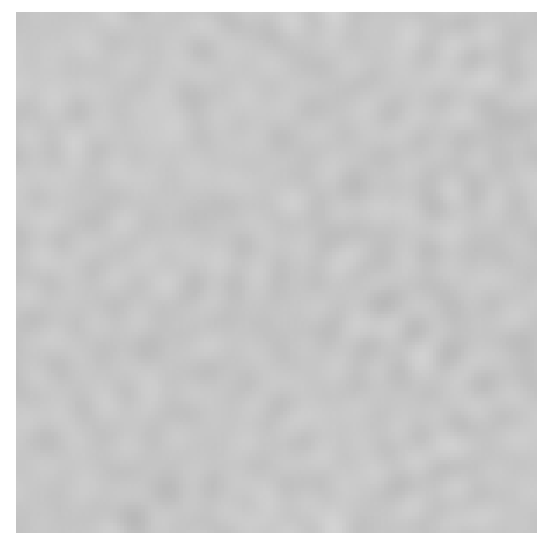

(c)

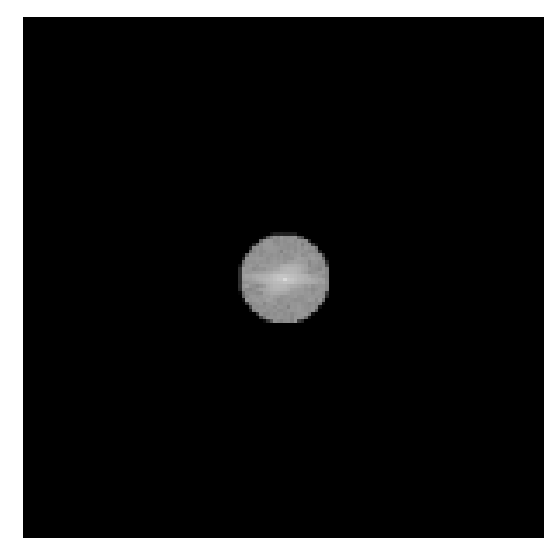

(b)

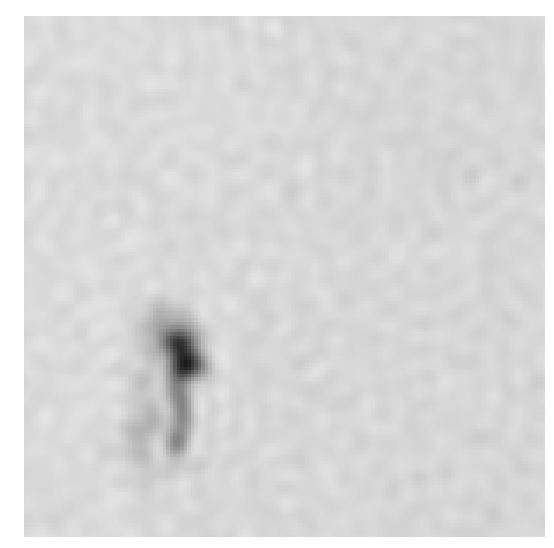

(d)

Figure 4. (a), (b) The respective Fourier domain images of Figures 2(a) and 2(b) with $F(u, v)=0$ for all frequency components outside the circle of radius $r_{\max }$; (c), (d) the restored images based on the power spectra in (a) and (b). 


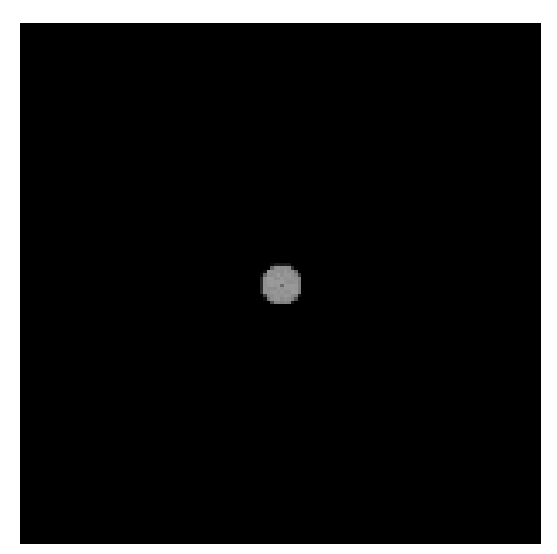

(a)

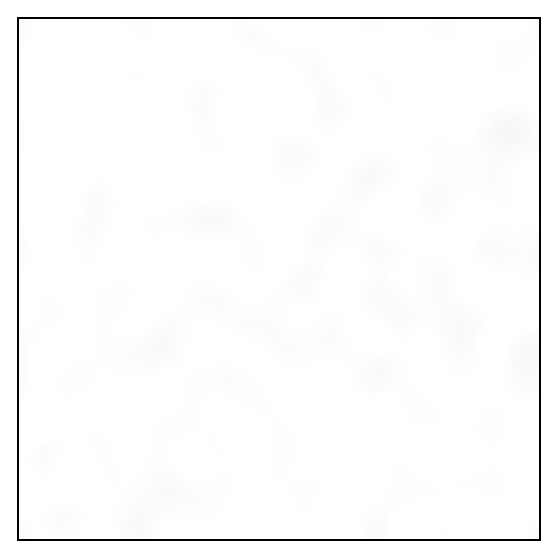

(c)

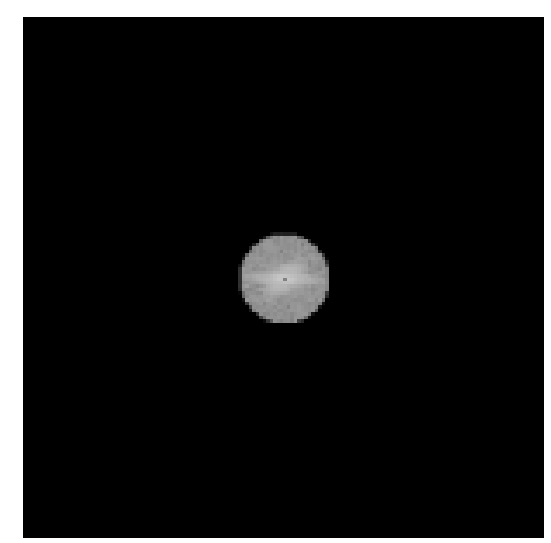

(b)

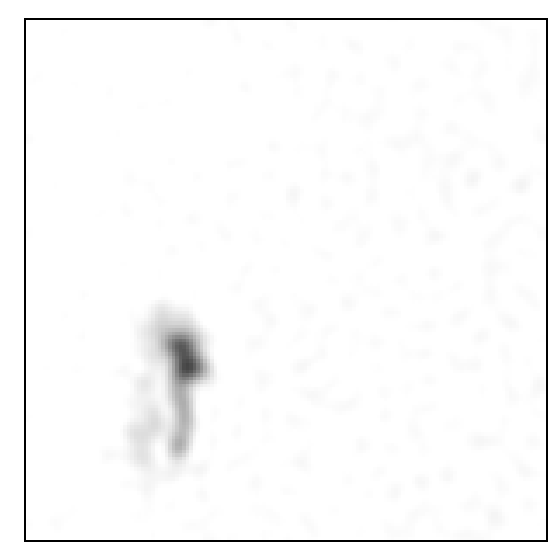

(d)

Figure 5. (a), (b) The respective Fourier domain images of Figures 2(a) and 2(b) with $F(u, v)=0$ for the central component and all frequency components outside the circle of radius $r_{\max }$; (c), (d) the restored images based on the power spectra in (a) and (b). 


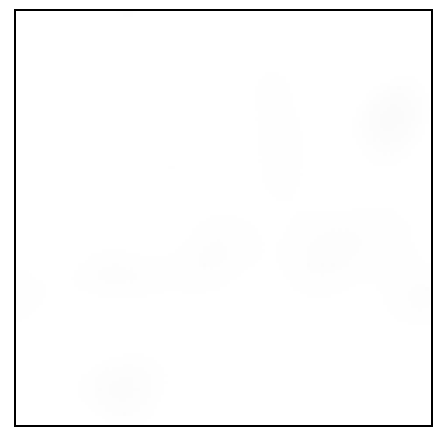

(a1) $r=5$

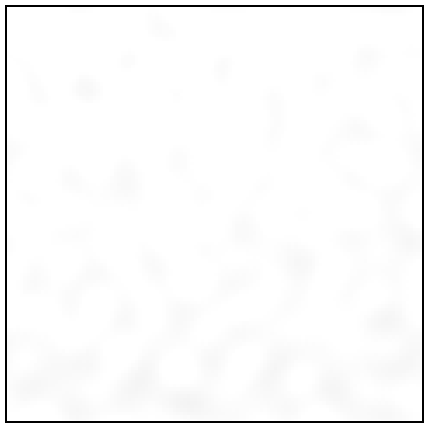

(a2) $r=10$

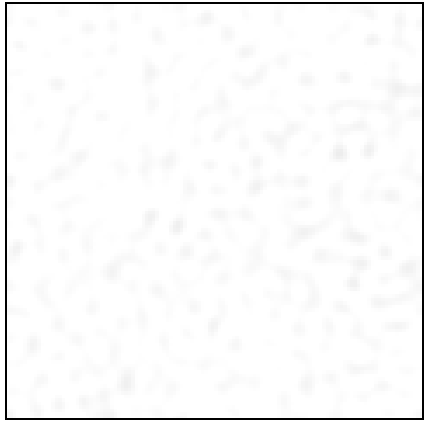

(a3) $r=20$

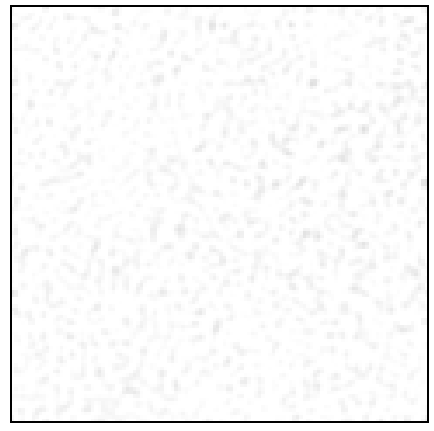

(a4) $r=40$

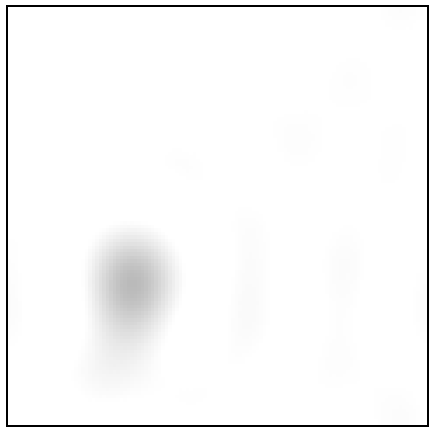

(b1) $r=5$

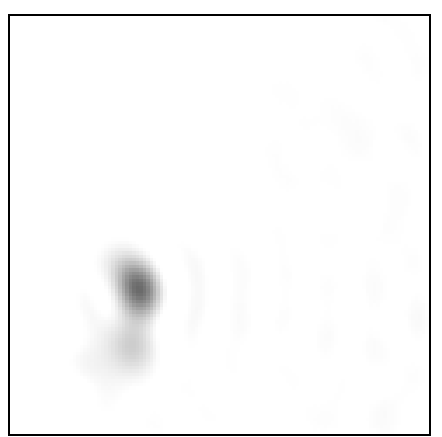

(b2) $r=10$

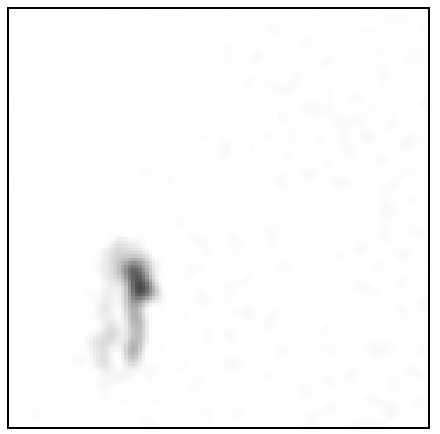

(b3) $r=20$

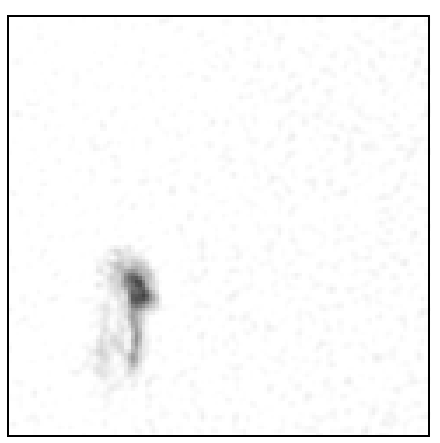

(b4) $r=40$

Figure 6. (a1)-(a4) The restored images from the circles of radii 5, 10, 20 and 40 pixels for the faultless sandpaper surface in Figure 2(a); (b1)-(b4) The restored images from the circles of radii 5, 10, 20 and 40 pixels for the defective sandpaper surface in Figure 2(b). 


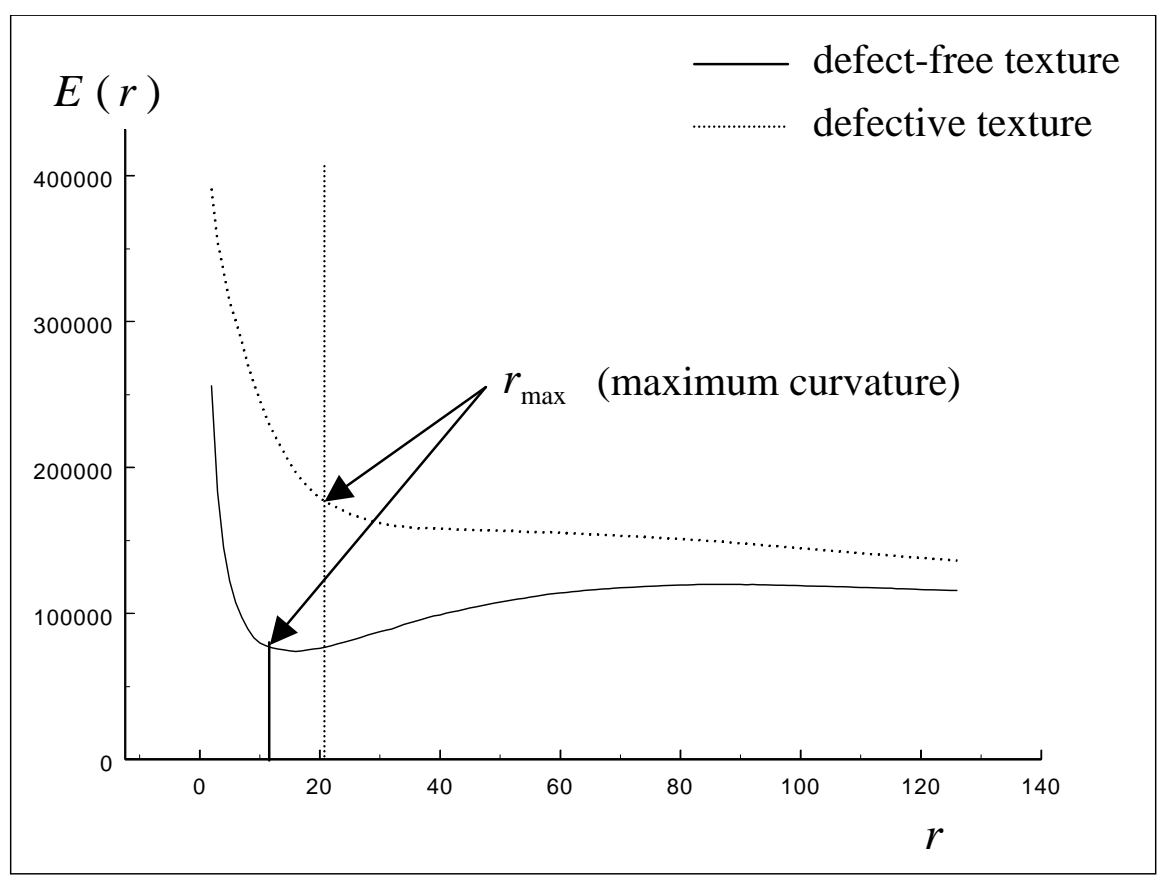

Figure 7. The plots of average power $E(r)$ as a function of radius $r$ for the sandpaper images in Figures 2(a) and (b). 


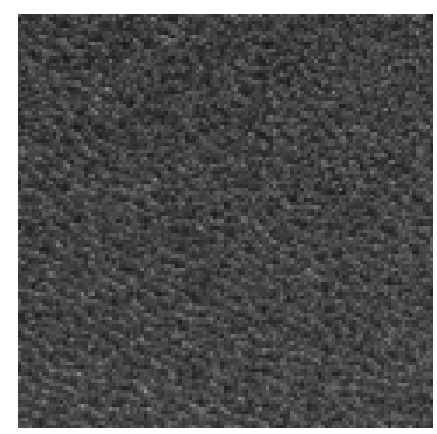

(a)

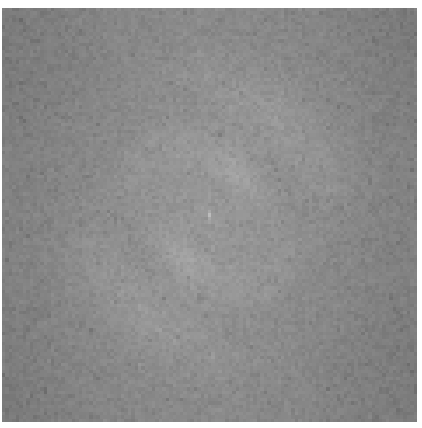

(c)

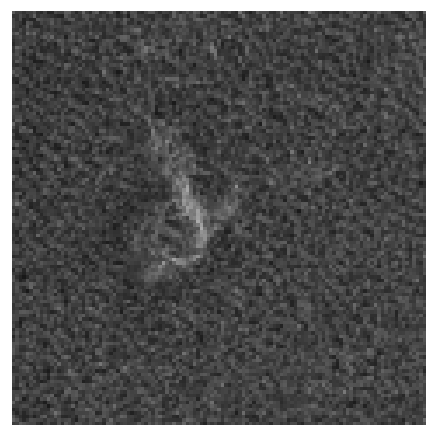

(b)

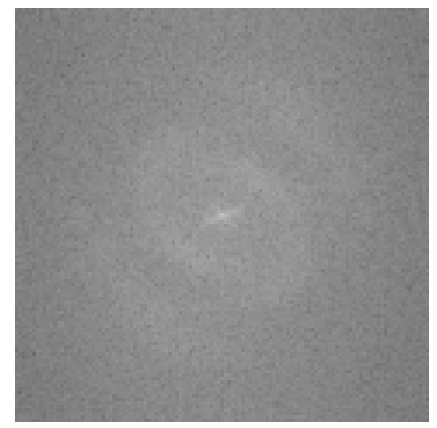

(d)

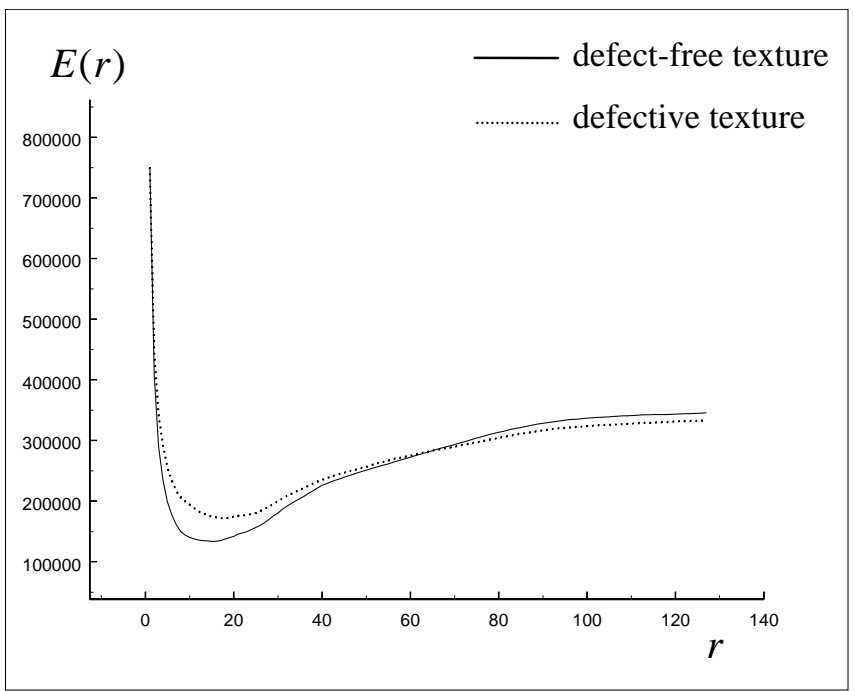

(e)

Figure 8. Two leather samples used for evaluating the sensitivity of changes in radius $r_{\max }$ : (a), (b) one faultless leather image and one defective version of the leather surface; (c), (d) the respective Fourier domain images; (e) the curves of average power $E(r)$ with respect to varying radius for (c) and (d). 


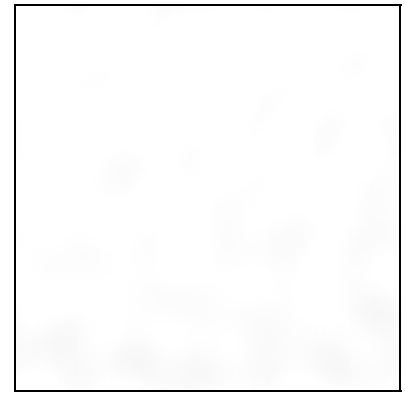

(a1) $r=7$

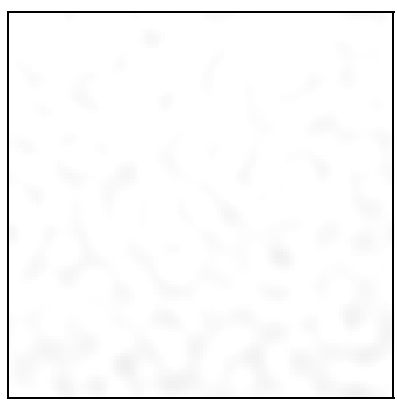

(a4) $r=13$

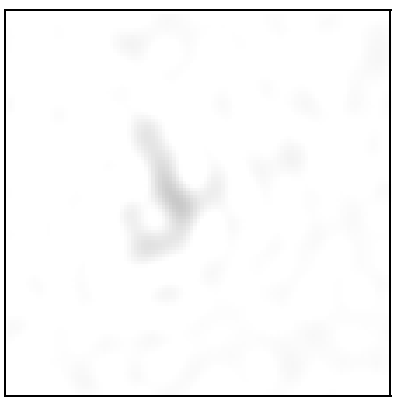

(b1) $r=10$

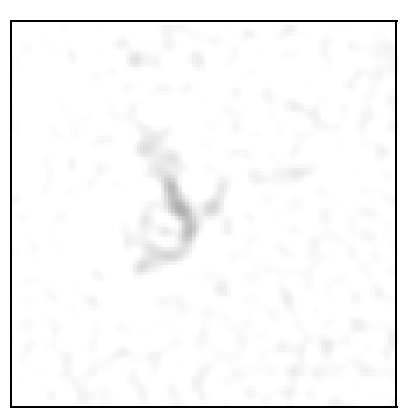

(b4) $r=20$

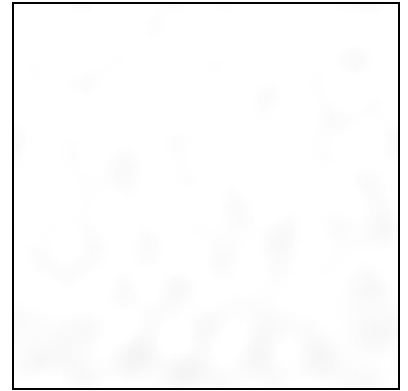

(a2) $r=9$

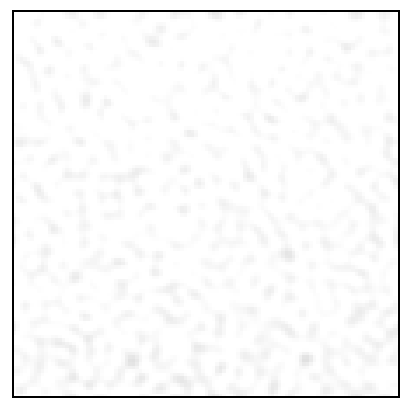

(a5) $r=25$

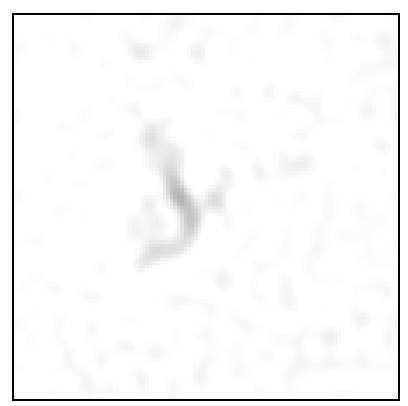

(b2) $r=16$

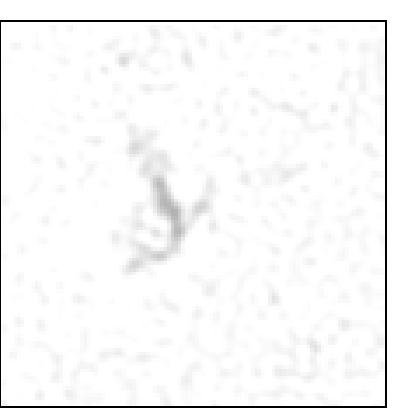

(b5) $r=25$

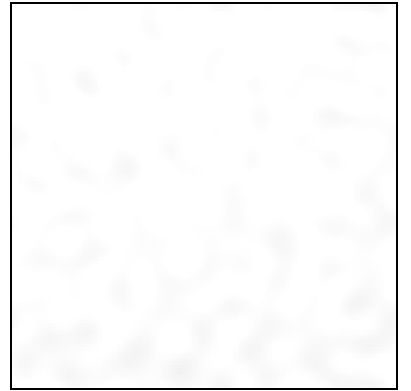

(a3) $r_{\max }=11$

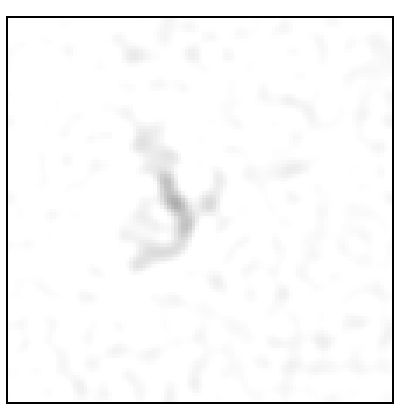

(b3) $r_{\max }=18$

Figure 9. (a1)-(a5) The restored images from the circles of radii 7, 9, 11, 13 and 25 pixels for the faultless leather surface in Figure 8(a); (b1)-(b5) the restored images from the circles of radii 10, 16, 18, 20 and 25 pixels for the defective leather surface in Figure 8(b). 


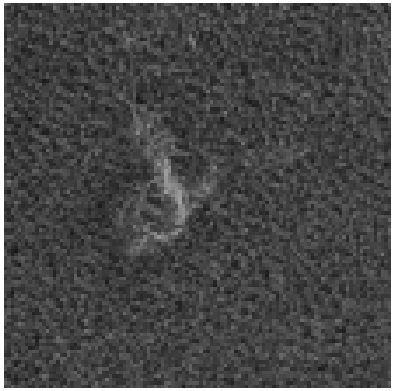

(a1)

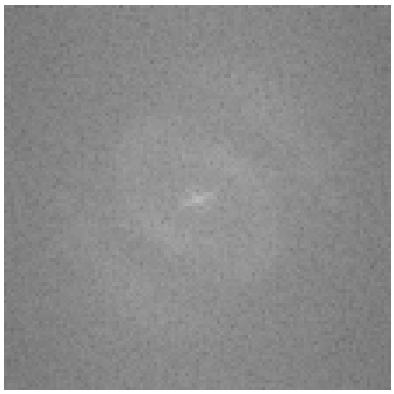

(a2)

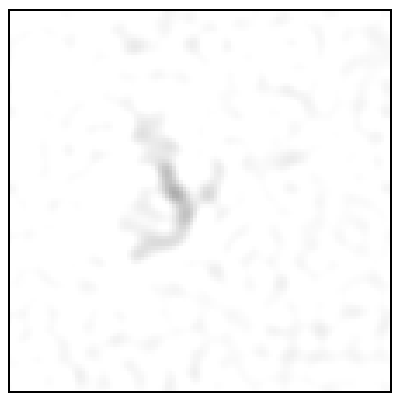

(a3) $r_{\max }=18$

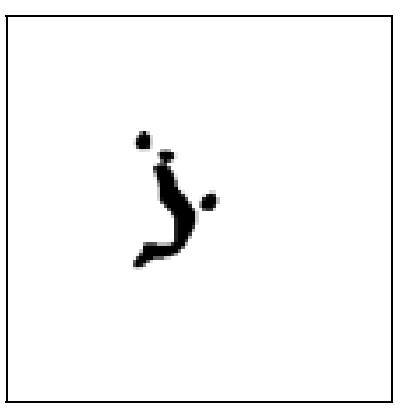

(a4)

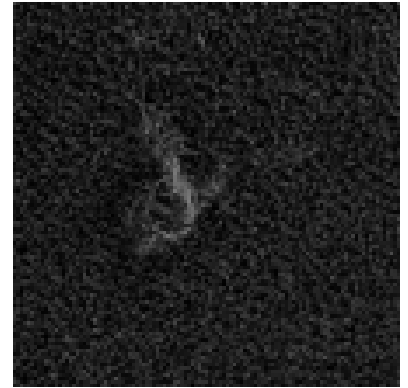

(b1)

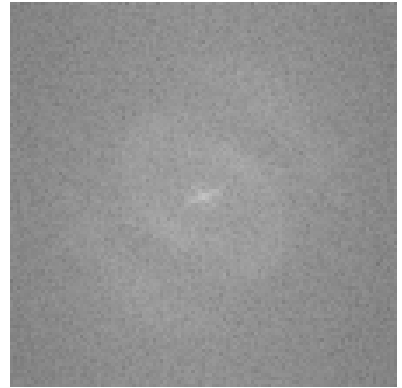

(b2)

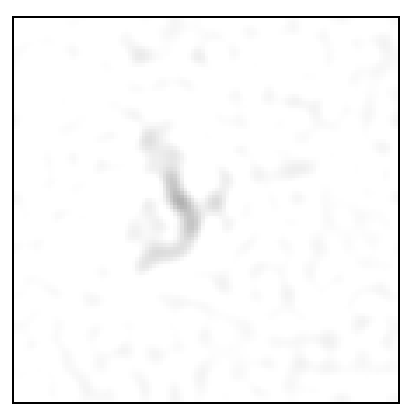

(b3) $r_{\max }=17$

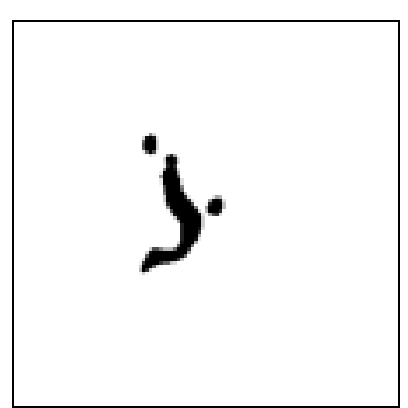

(b4)

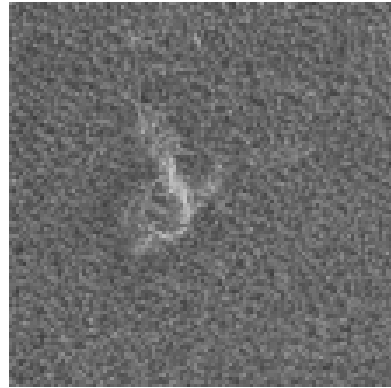

$(\mathrm{c} 1)$

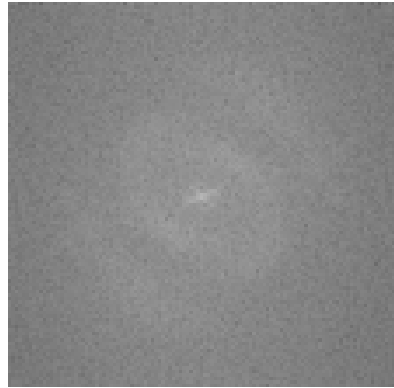

(c2)

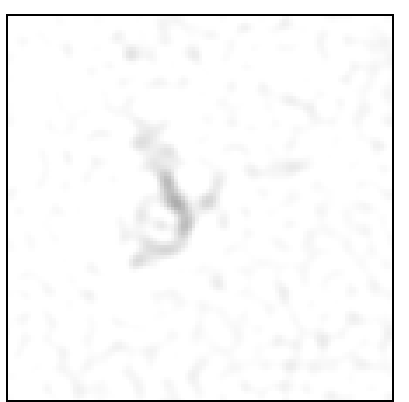

(c3) $r_{\max }=20$

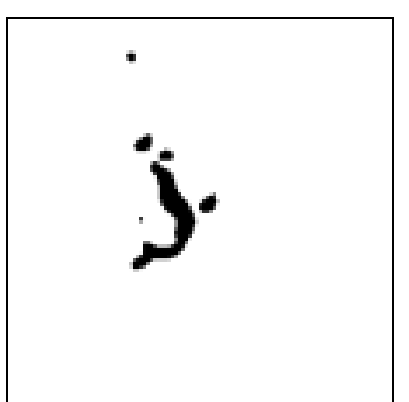

(c4)

Figure 10. The effect of changes in illumination intensity: (a1)-(c1) a defective leather surface under regular, underexposed and overexposed lighting conditions; (a2)-(c2) the respective power spectra; (a3)-(c3) the respective restored images; (a4)-(c4) the binarized images that show the defects in black. 


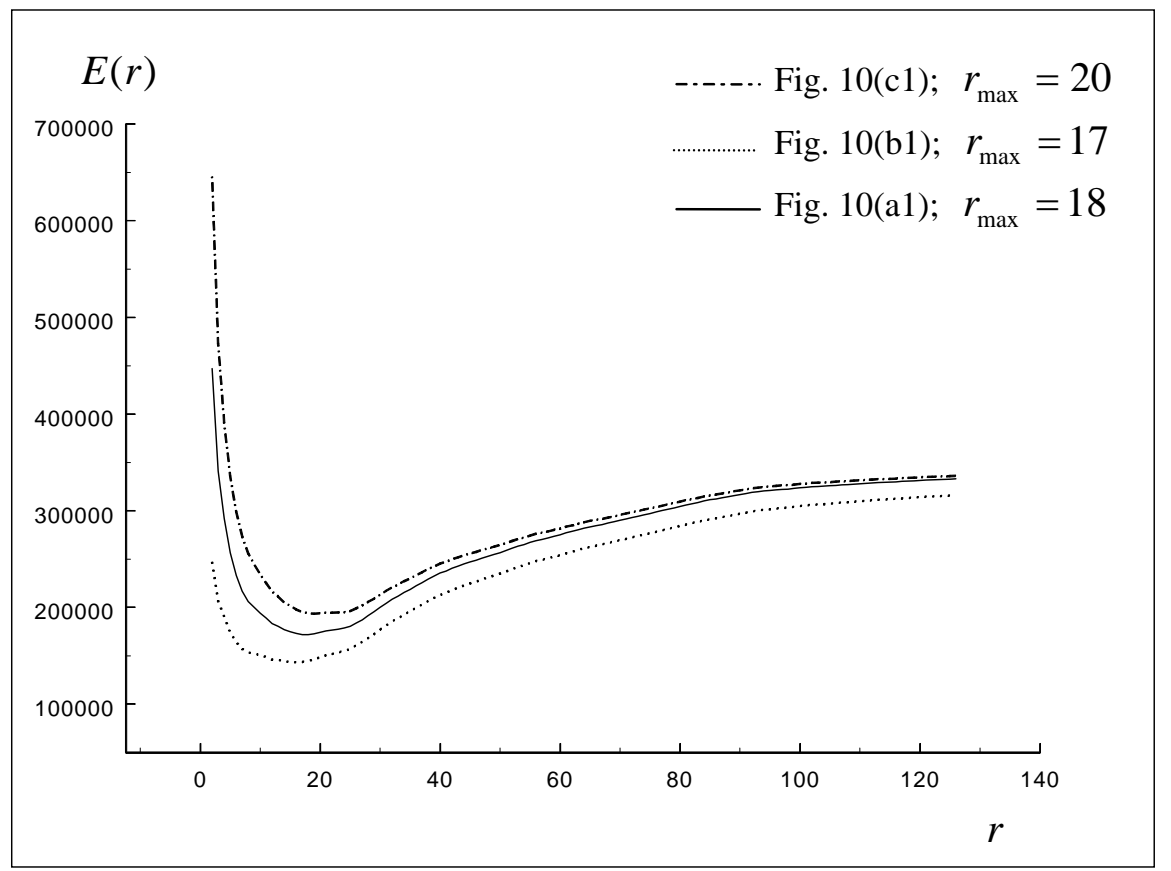

Figure 11. The plots of $E(r)-r$ curves for the leather images in Figures 10(a1)-(c1). 


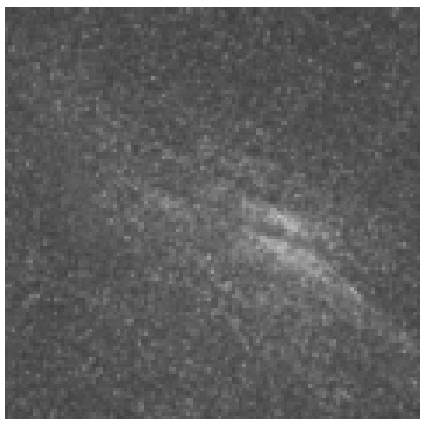

(a)

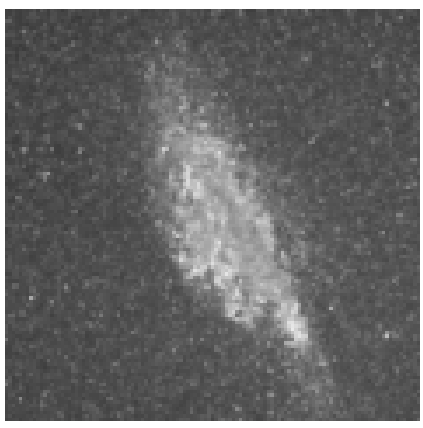

(b)

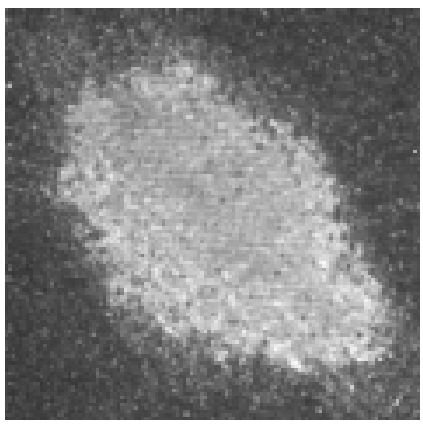

(c)

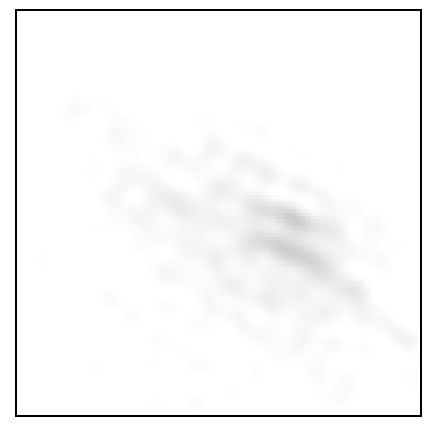

(d)

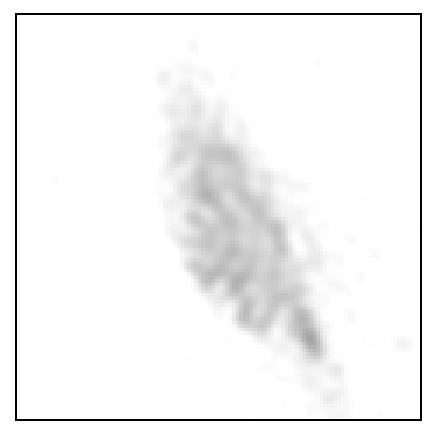

(e)

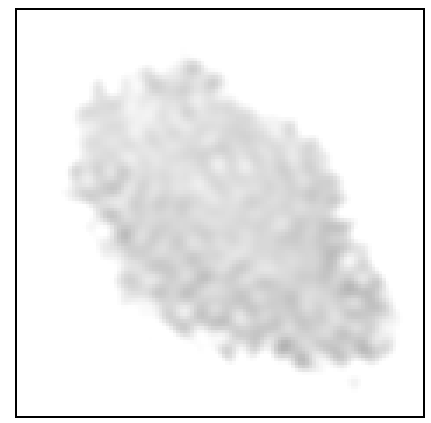

(f)

Figure 12. The effect of changes in size for defects in fine-grain sandpaper: (a), (b), (c) sandpaper images containing increasing sizes of defects; (d), (e), (f) the respective restored images. They show that all defects are well retained in the resulting images. 


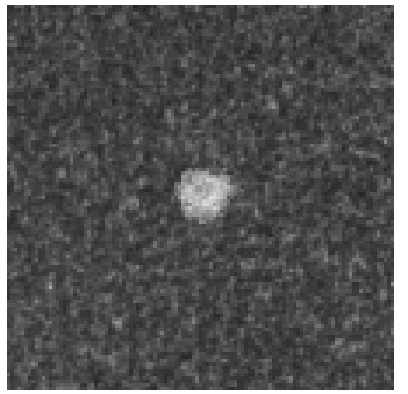

(a)

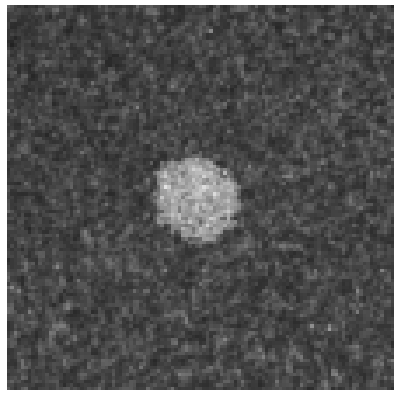

(b)

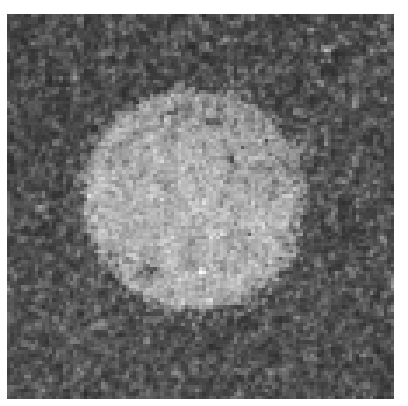

(c)

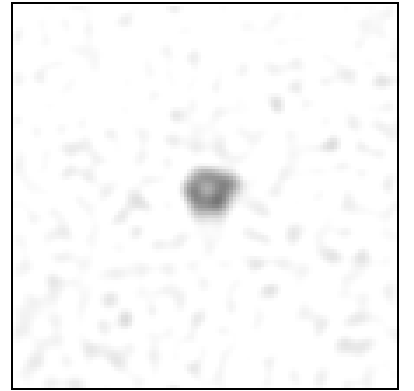

(d)

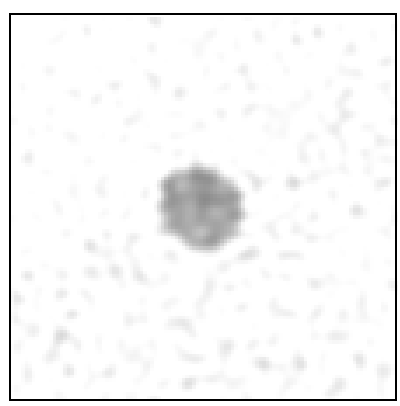

(e)

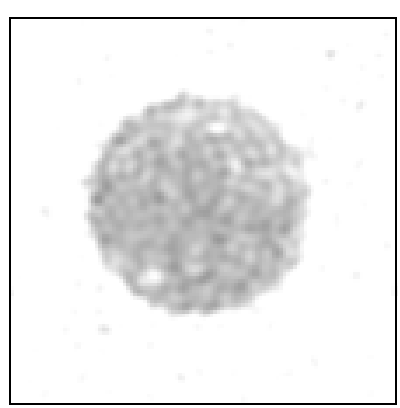

(f)

Figure 13. The effects of defect sizes in coarse-grain sandpaper: (a), (b), (c) sandpaper images containing increasing sizes of defects; (d), (e), (f) the respective restored images. 


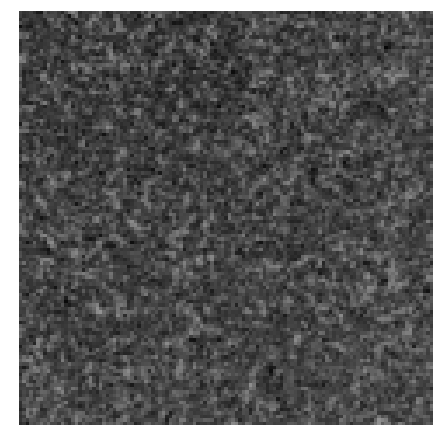

(a1)

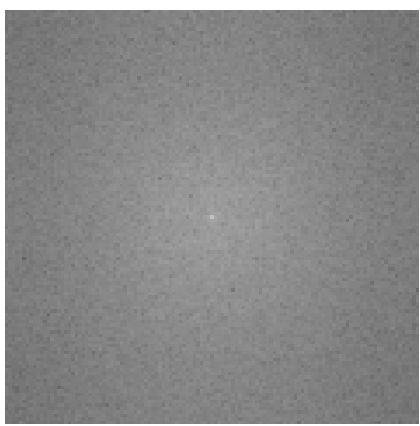

(a2)

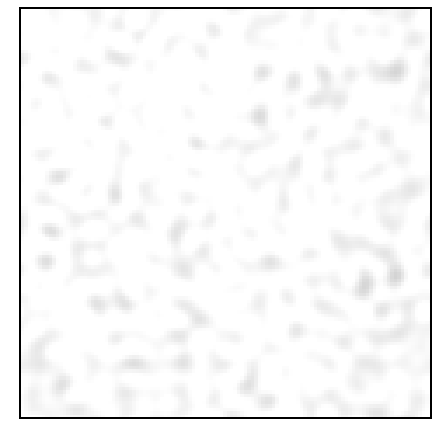

(a3)

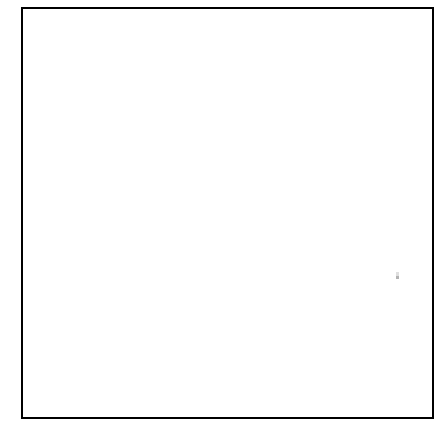

(a4)

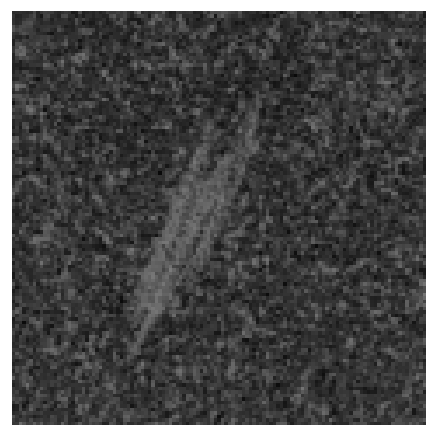

(b1)

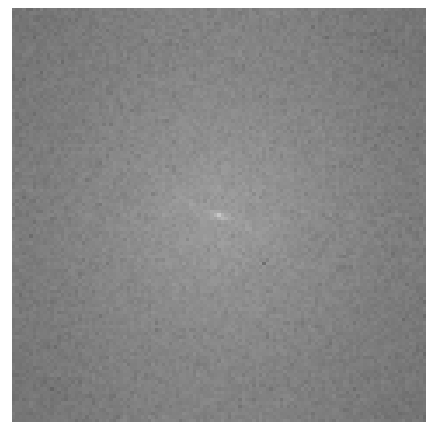

(b2)

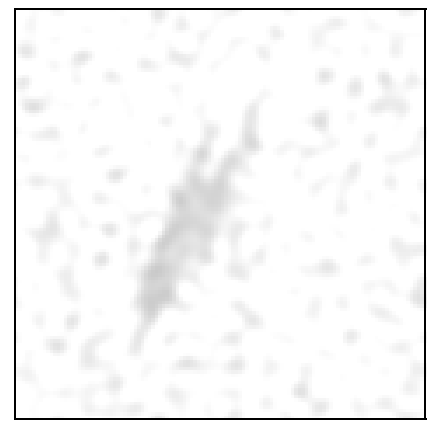

(b3)

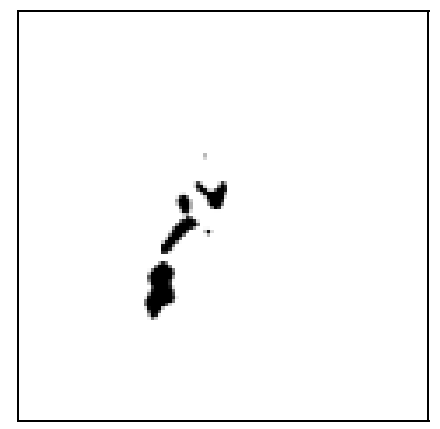

(b4)

Figure 14. (a1) A clear cast surface; (b1) a defective cast surface with scratches; (a2), (b2) the respective power spectra; (a3), (b3) the respective reconstruction results; (a4), (b4) the resulting binary images for defect segmentation. 


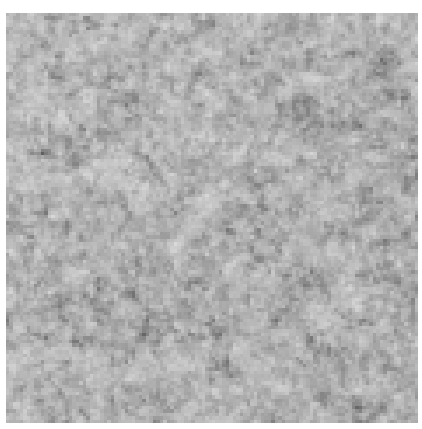

(a1)

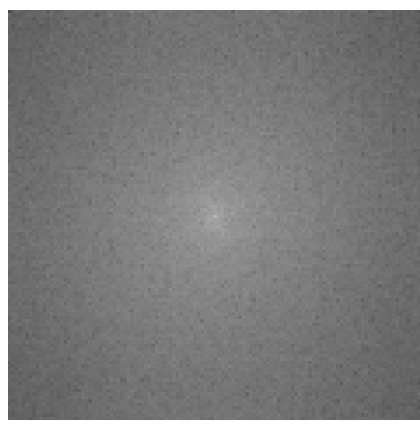

(a2)

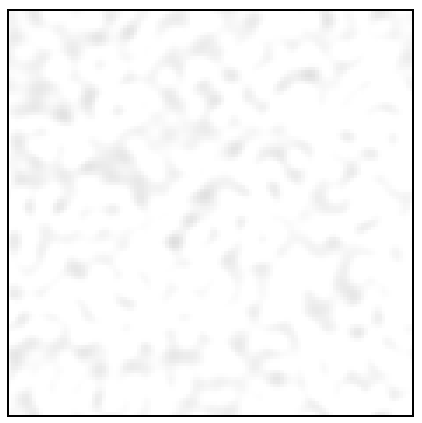

(a3)

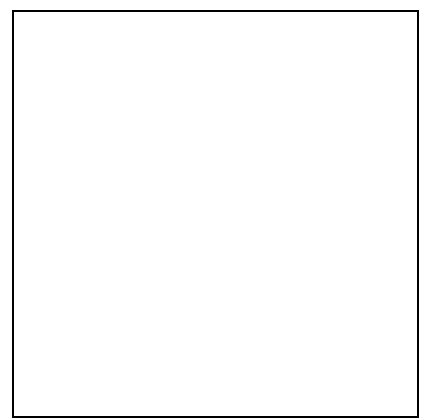

(a4)

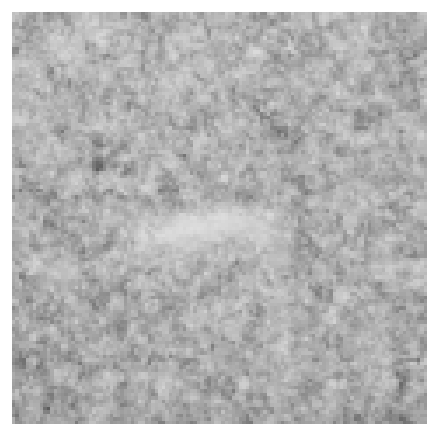

(b1)

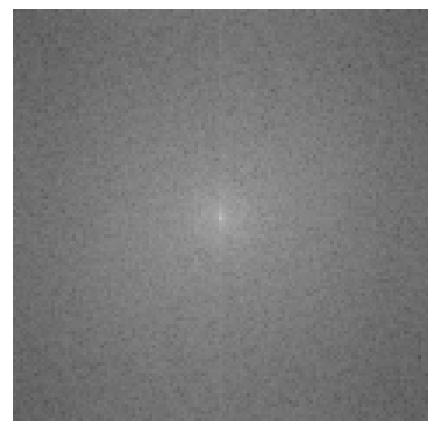

(b2)

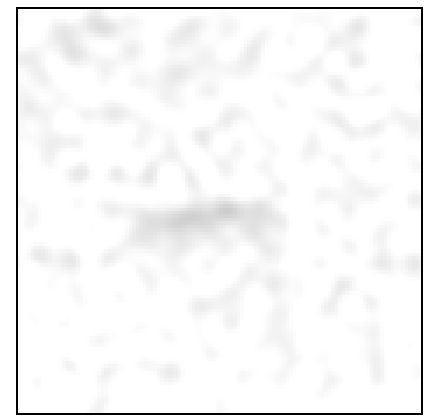

(b3)

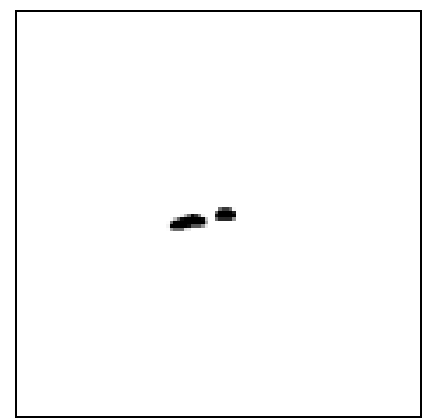

(b4)

Figure 15. (a1) A faultless wool surface; (b1) a defective wool surface; (a2), (b2) the respective power spectra; (a3), (b3) the respective restored images; (a4), (b4) the resulting binary images. 


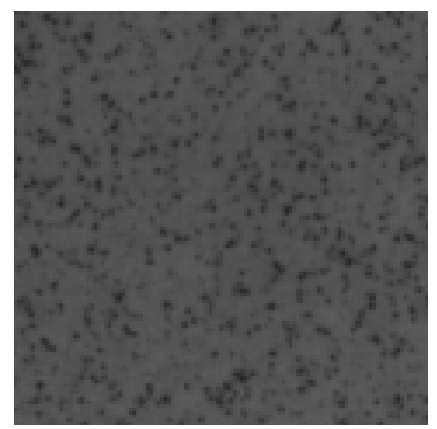

(a1)

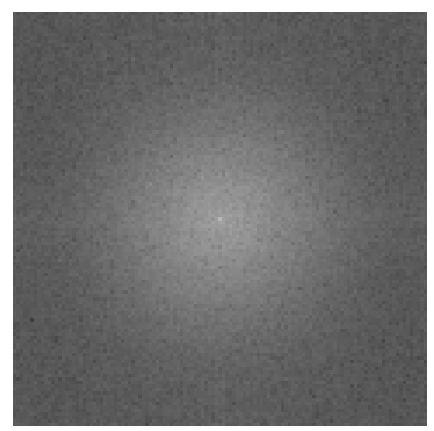

(a2)

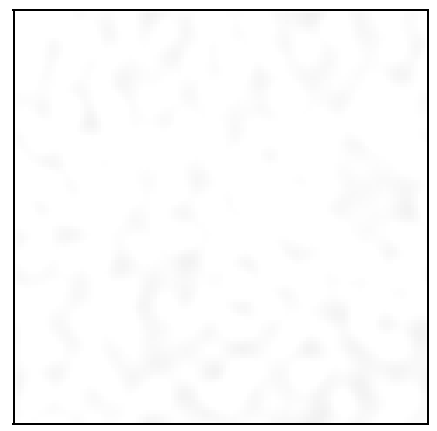

(a3)

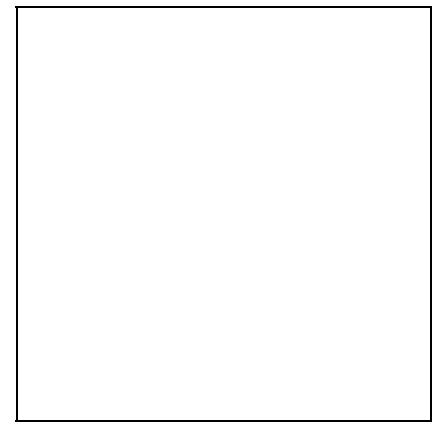

(a4)

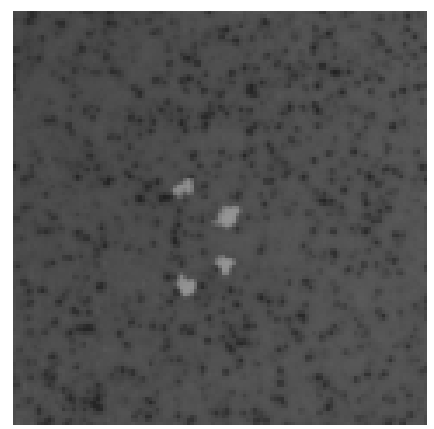

(b1)

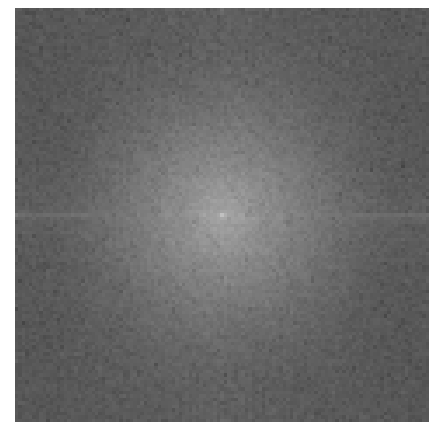

(b2)

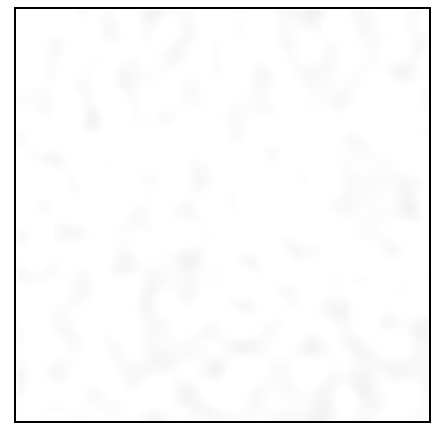

(b3)

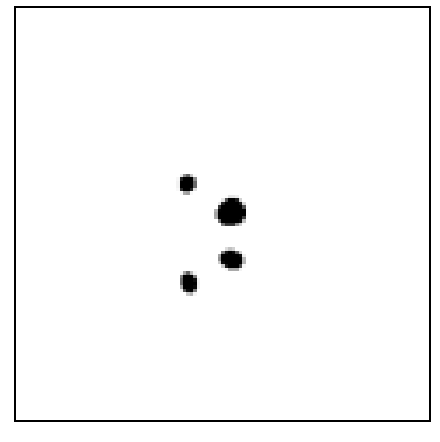

(b4)

Figure 16. (a1) A faultless tile surface; (b1) a defective tile surface; (a2), (b2) the respective power spectra; (a3), (b3) the respective restored images; (a4), (b4) the resulting binary images. 


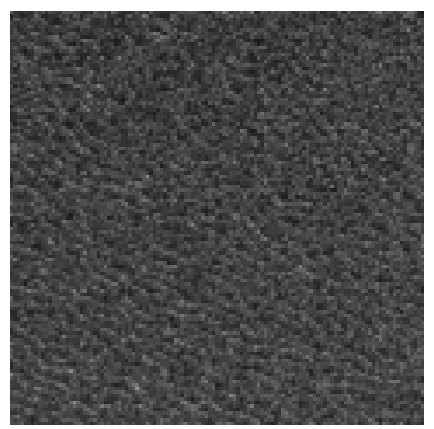

(a1)

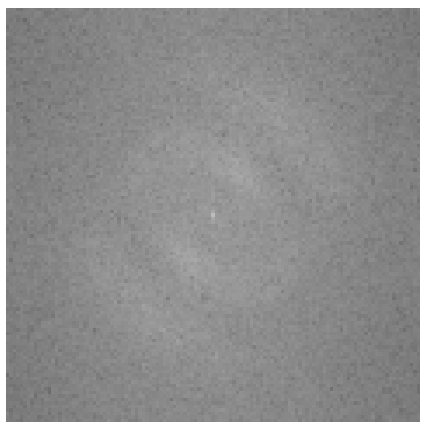

(a2)

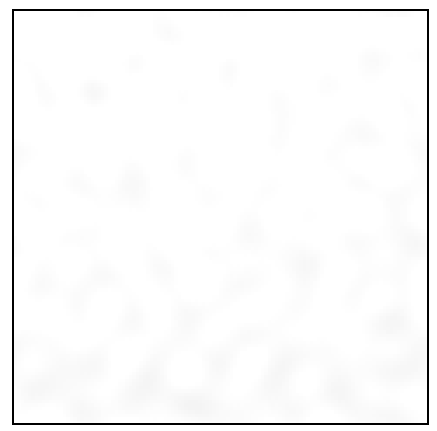

(a3)

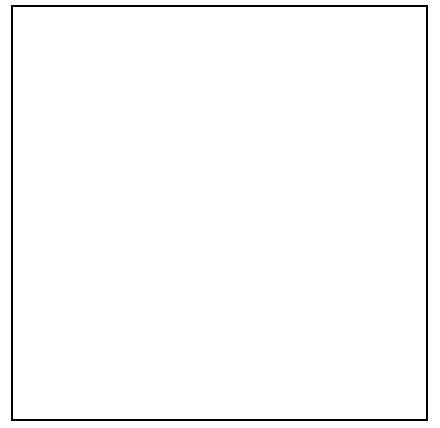

(a4)

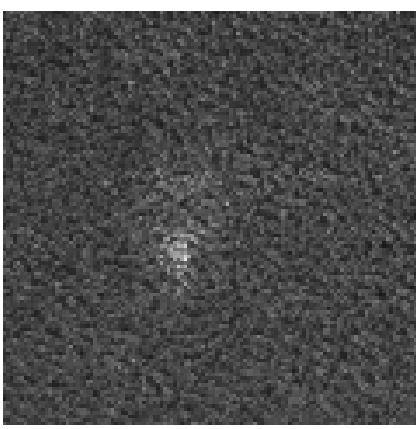

(b1)

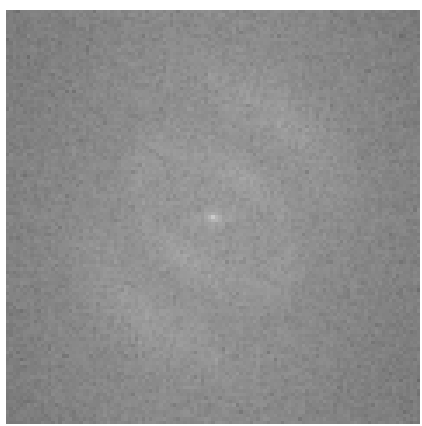

(b2)

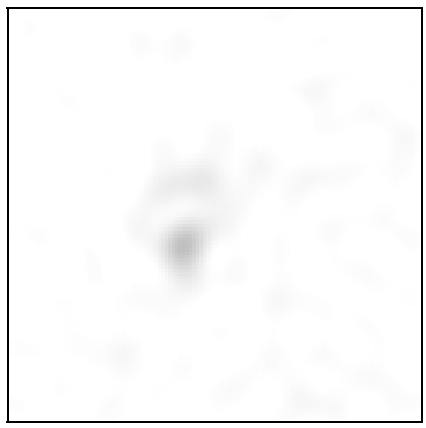

(b3)

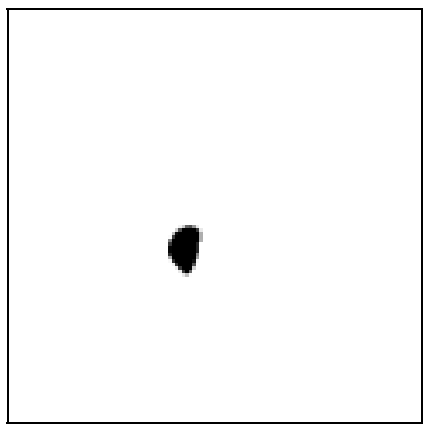

(b4)

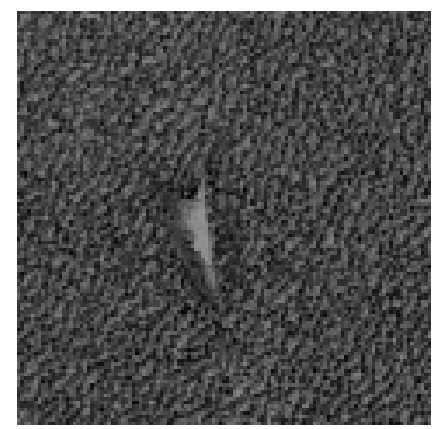

(c1)

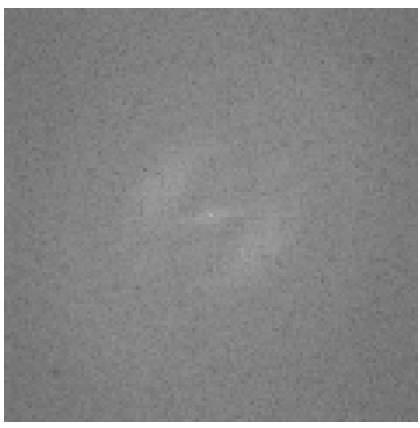

(c2)

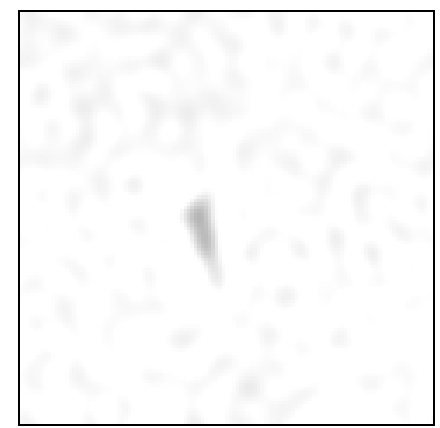

(c3)

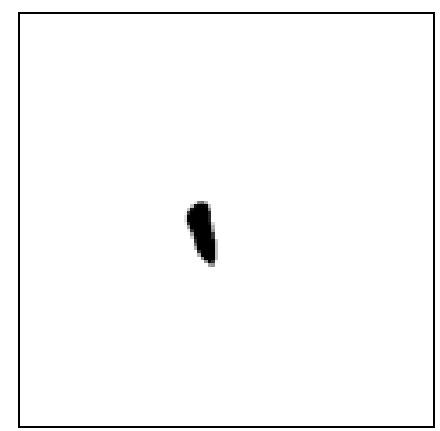

(c4)

Figure 17. (a1) A clear leather surface; (b1) the leather surface with a wear defect; (c1) the leather surface with a scratch; (a2)-(c2) the respective power spectra; (a3)-(c3) the respective restored images; (a4)-(c4) the resulting binary images that show the detected defects in black. 


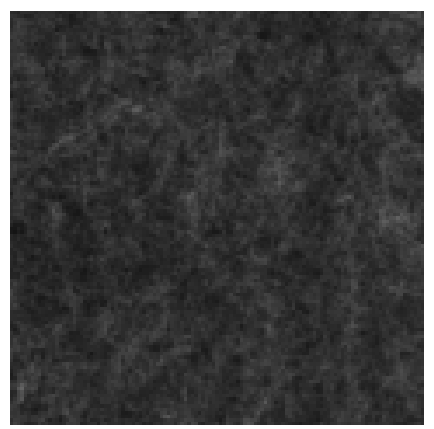

(a1)

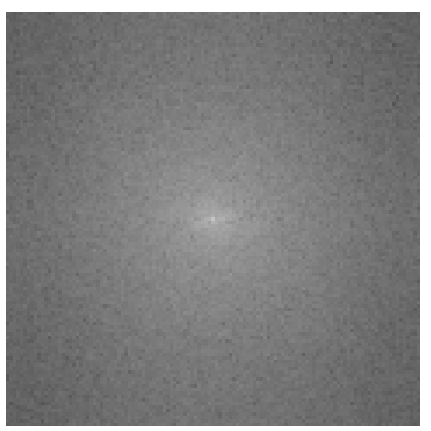

(a2)

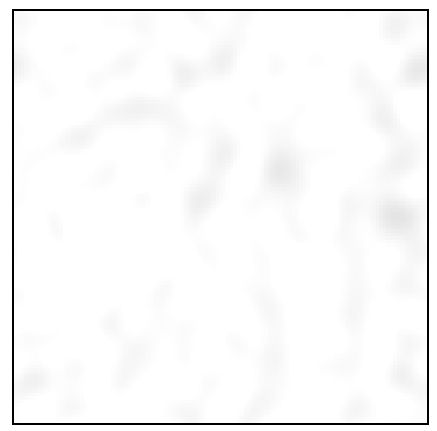

(a3)

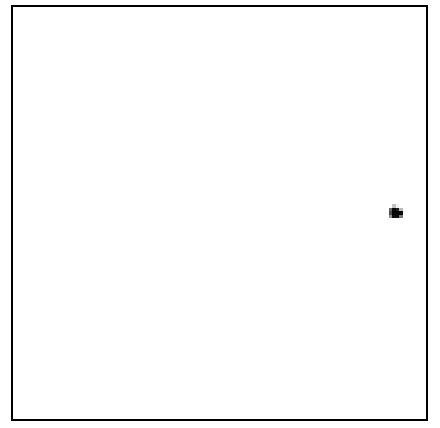

(a4)

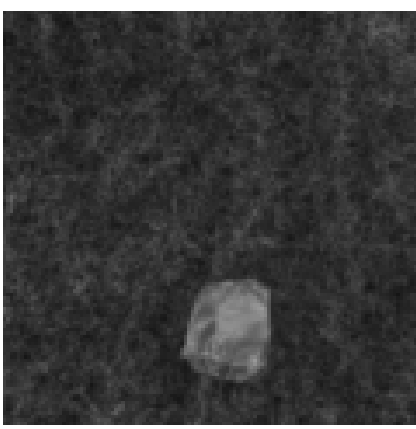

(b1)

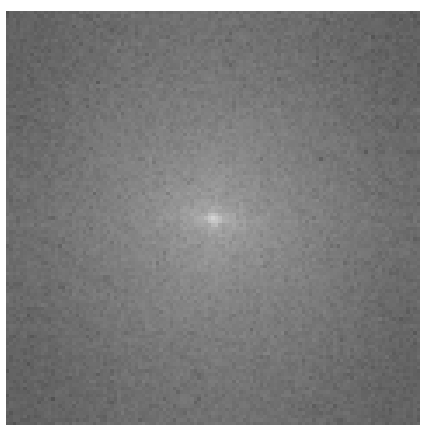

(b2)

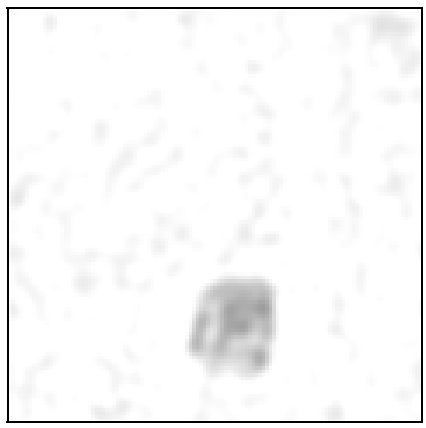

(b3)

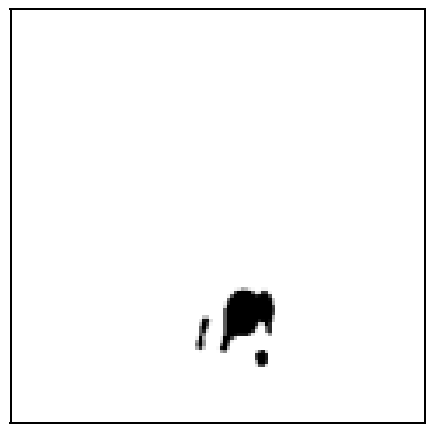

(b4)

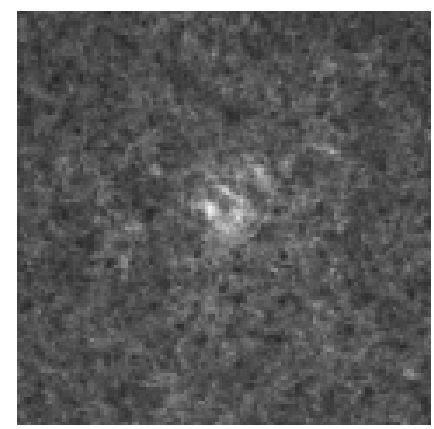

(c1)

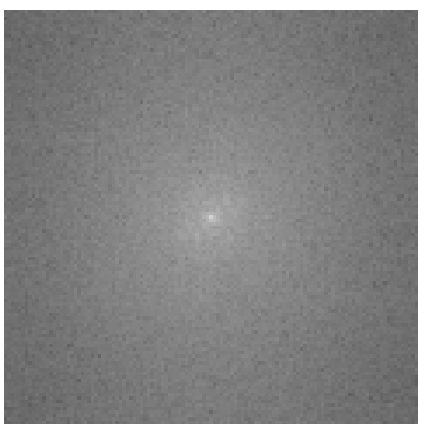

(c2)

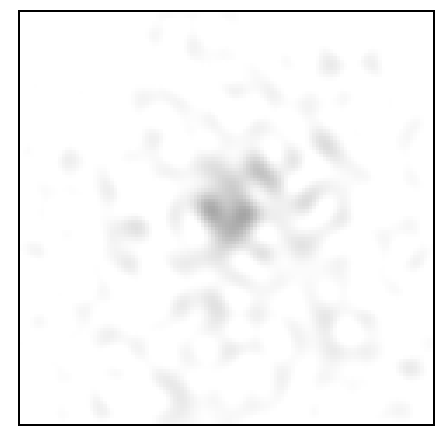

(c3)

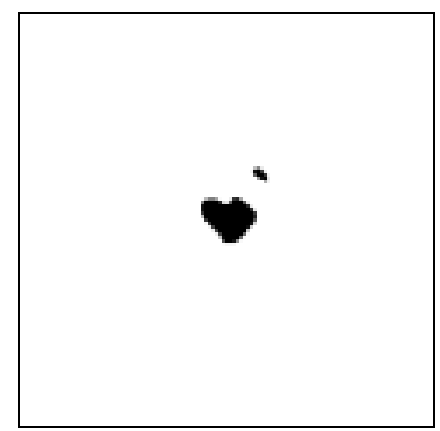

(c4)

Figure 18. (a1) A faultless carpet surface; (b1), (c1) two defective carpet surfaces; (a2)-(c2) the respective power spectra; (a3)-(c3) the respective restored images; (a4)-(c4) the resulting binary images. 


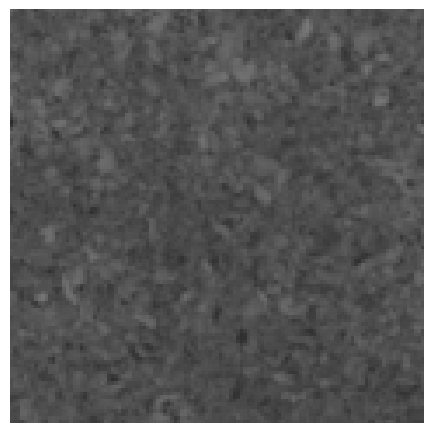

(a1)

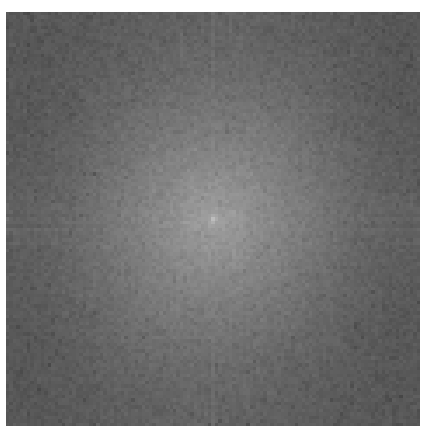

(a2)

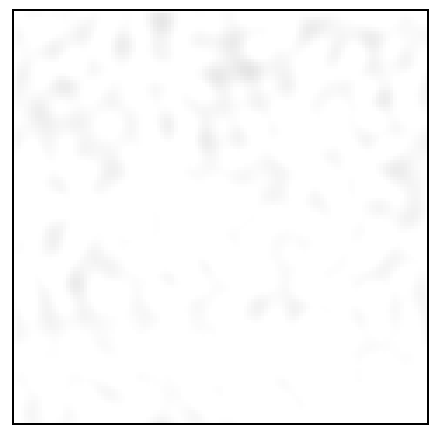

(a3)

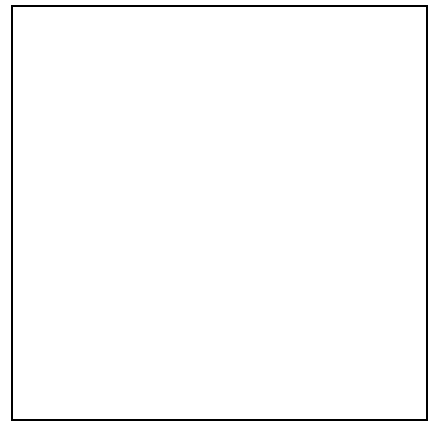

(a4)

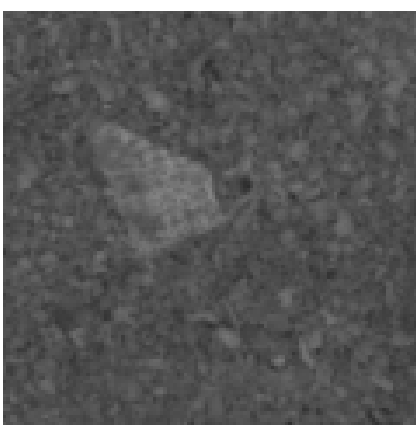

(b1)

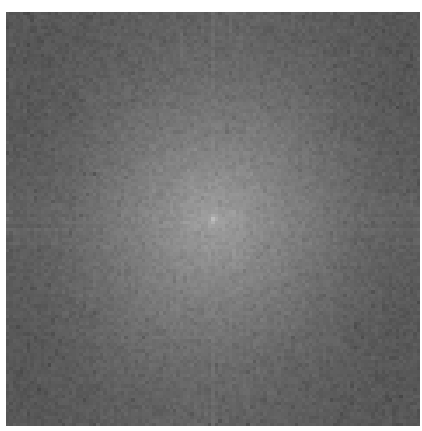

(b2)

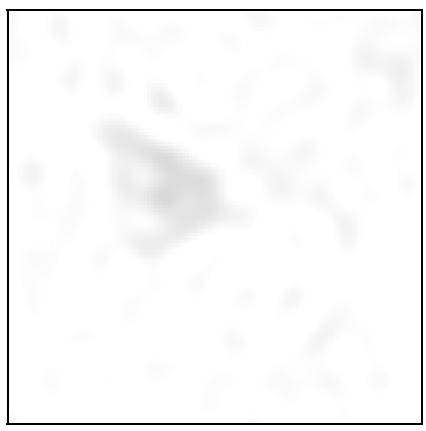

(b3)

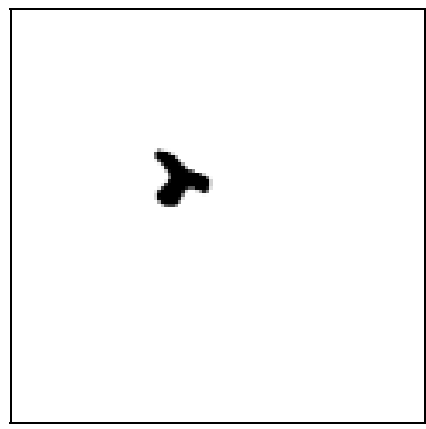

(b4)

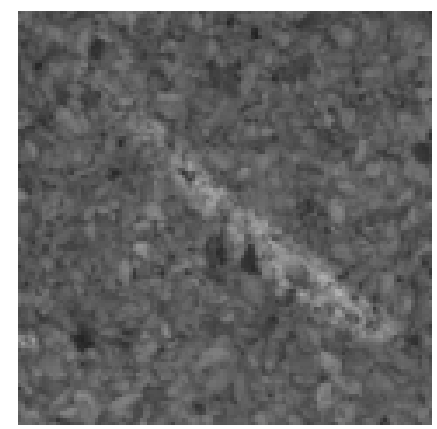

(c1)

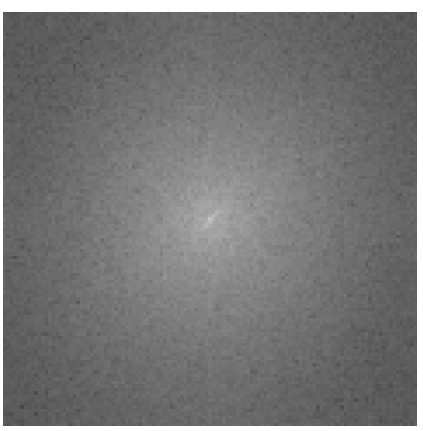

(c2)

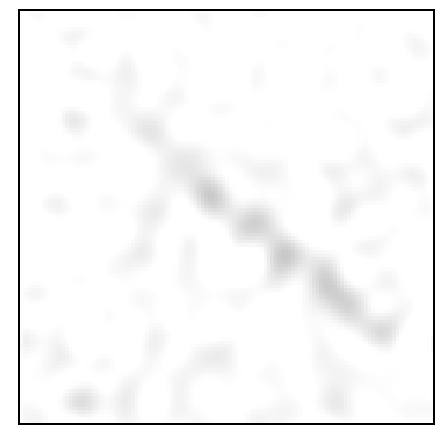

(c3)

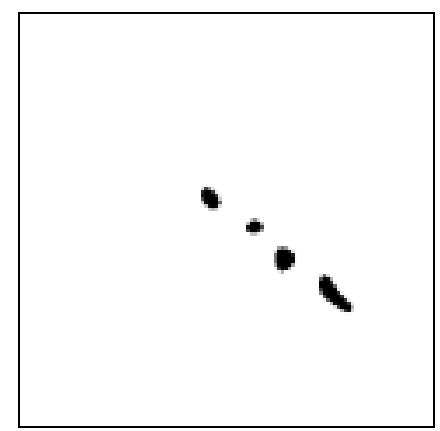

(c4)

Figure 19. (a1) A faultless cork surface; (b1), (c1) two defective cork surfaces; (a2)-(c2) the respective power spectra; (a3)-(c3) the respective restored images; (a4)-(c4) the resulting binary images. 


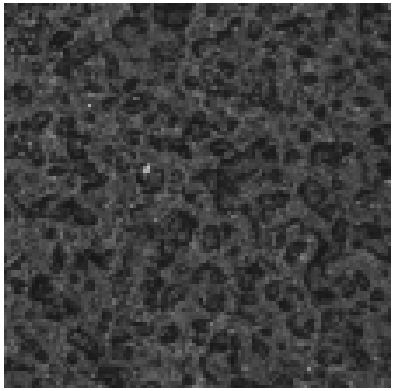

(a1)

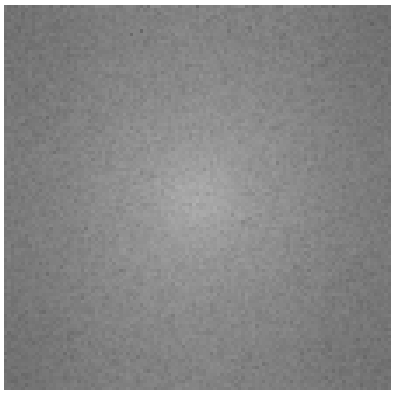

(a2)

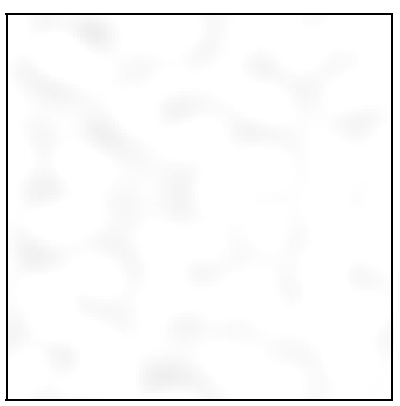

(a3)

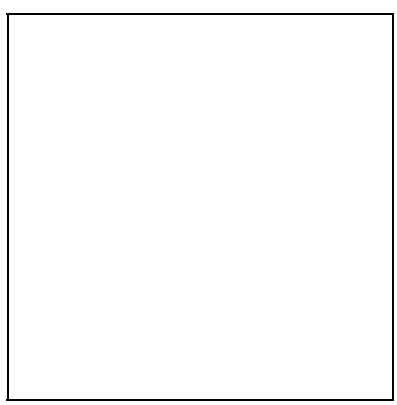

(a4)

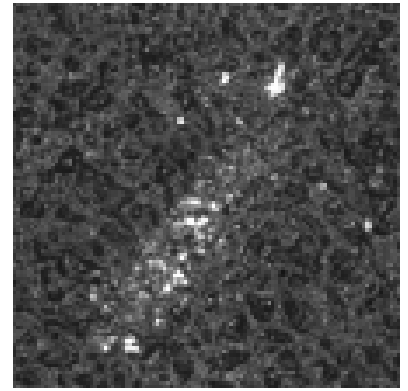

(b1)

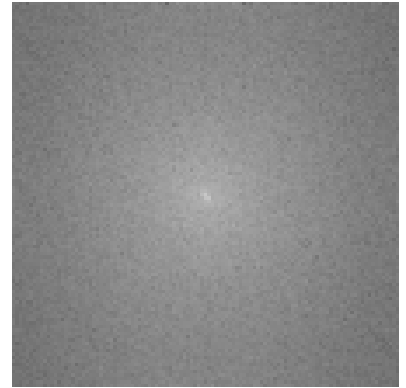

(b2)

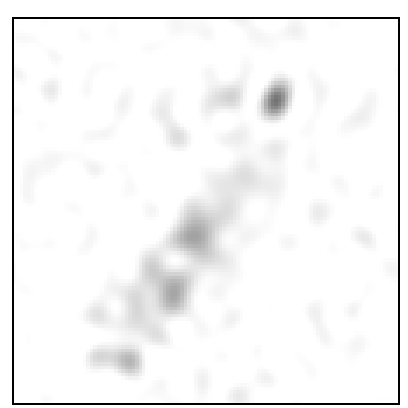

(b3)

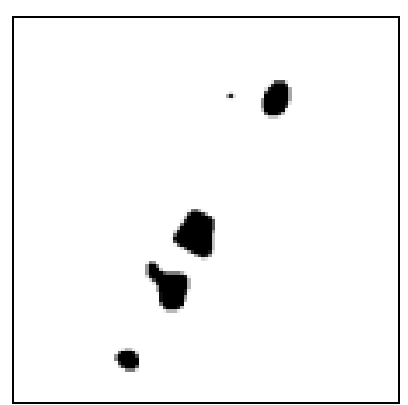

(b4)

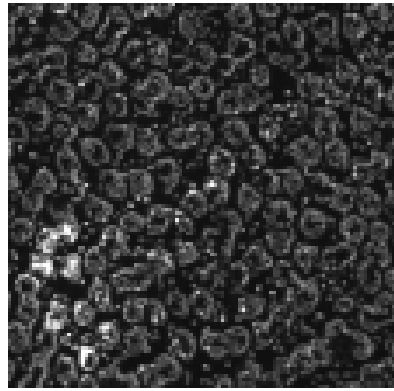

(c1)

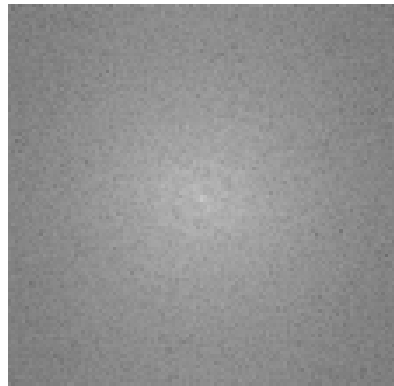

(c2)

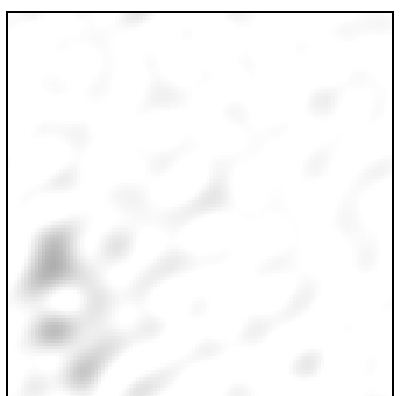

(c3)

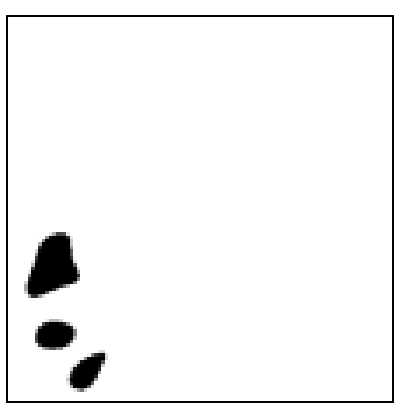

(c4)

Figure 20. (a1) A faultless coarse-grain sandpaper surface; (b1), (c1) two defective versions of the sandpaper; (a2)-(c2) the respective power spectra; (a3)-(c3) the respective restored images; (a4)-(c4) the resulting binary images. 